TRANSACTIONS OF THE

AMERICAN MATHEMATICAL SOCIETY

Volume 359, Number 8, August 2007, Pages 3647-3668

S 0002-9947(07)04040-8

Article electronically published on March 7, 2007

\title{
SEMI-EDGES, REFLECTIONS AND COXETER GROUPS
}

\author{
RALF GRAMLICH, GEORG W. HOFMANN, AND KARL-HERMANN NEEB
}

\begin{abstract}
We combine the theory of Coxeter groups, the covering theory of graphs introduced by Malnic, Nedela and Skoviera and the theory of reflections of graphs in order to obtain the following characterization of a Coxeter group:

Let $\pi: \Gamma \rightarrow(v, D, \iota,-1)$ be a 1-covering of a monopole admitting semiedges only. The graph $\Gamma$ is the Cayley graph of a Coxeter group if and only if $\pi$ is regular and any deck transformation in $\Delta(\pi)$ that interchanges two neighboring vertices of $\Gamma$ acts as a reflection on $\Gamma$.
\end{abstract}

\section{INTRODUCTION}

There are numerous similarities between the covering theory of graphs and the covering theory of topological spaces. In algebraic topology, cf. e.g. Definition III.3.1 of [3], a map $p: X \rightarrow Y$ between arcwise connected, locally arcwise connected Hausdorff spaces is called a covering map if each element $y \in Y$ has an arcwise connected neighborhood $U$ such that $p^{-1}(U)$ is a nonempty disjoint union of sets $U_{\alpha}$ on which $p_{\mid U_{\alpha}}$ is a homeomorphism $U_{\alpha} \stackrel{\approx}{\approx} U$. In classical graph theory one replaces the topological spaces $X$ and $Y$ by connected graphs $\Gamma_{1}$ and $\Gamma_{2}$, the neighborhood $U$ of a vertex $\mathbf{y} \in \Gamma_{2}$ by the induced subgraph $\Gamma_{2}(\mathbf{y})$ on the neighbors of $\mathbf{y}$ in $\Gamma_{2}$ and requires that for each preimage $\mathbf{x}$ in $\Gamma_{1}$ of $\mathbf{y}$ the restriction of $p$ to $\Gamma_{1}(\mathbf{x})$ is a bijection, resp. isomorphism, onto $\Gamma_{2}(\mathbf{y})$.

More precisely, a surjection $\pi: \Gamma_{1} \rightarrow \Gamma_{2}$ between connected graphs $\Gamma_{1}, \Gamma_{2}$ is called a 1-covering map if for each $\mathbf{x}$ in $\Gamma_{1}$ the map $\pi$ induces a bijection between the set of vertices of $\Gamma_{1}(\mathbf{x})$ onto the set of vertices of $\Gamma_{2}\left(\mathbf{x}^{\pi}\right)$. On the other hand, a surjection $\pi: \Gamma_{1} \rightarrow \Gamma_{2}$ between connected graphs $\Gamma_{1}, \Gamma_{2}$ is called a 2 -covering map if for each $\mathbf{x}$ in $\Gamma_{1}$ the map $\pi$ induces a graph isomorphism between the induced subgraph $\Gamma_{1}(\mathbf{x})$ onto the induced subgraph $\Gamma_{2}\left(\mathbf{x}^{\pi}\right)$.

The notion of 1-coverings (or local isomorphisms, as they are sometimes called) is important for the study of epimorphisms between point-line geometries under which the point row of each line is mapped bijectively onto the point row of its image and, dually, the line pencil of each point is mapped bijectively onto the line pencil of its image. In the case of flag-transitive point-line geometries this corresponds to the study of injective completions of amalgams consisting of two groups (the point

Received by the editors October 27, 2004 and, in revised form, March 22, 2005.

2000 Mathematics Subject Classification. Primary 05C25, 20F55, 55U10.

Key words and phrases. Coxeter groups, coverings of graphs, reflections on graphs, voltage spaces.

(C)2007 American Mathematical Society 
stabilizer and the line stabilizer). The geometry of the dihedral group $D_{2 n}=$ $\left\langle a, b \mid a^{2}=b^{2}=(a b)^{n}=1\right\rangle$ probably is the easiest example. A $2 n$-gon admits 1 covers by any $2 k n$-gon, $k \geq 1$; this 1-covering corresponds to the group epimorphism $D_{2 k n}=\left\langle a, b \mid a^{2}=b^{2}=(a b)^{k n}=1\right\rangle \rightarrow D_{2 n}=\left\langle a, b \mid a^{2}=b^{2}=(a b)^{n}=1\right\rangle$ induced by factoring out the cyclic normal subgroup generated by all $n$th powers of the product $a b$. Of course, the universal 1-cover of a $2 n$-gon is the tree of valency two, which corresponds to $D_{2 \infty}=\left\langle a, b \mid a^{2}=b^{2}=1\right\rangle$ being the universal completion of the amalgam $\langle a\rangle \cup\langle b\rangle$. For a thorough treatment of the relationship between covers of graphs and amalgams we refer the reader to [5], [6], 16, [17. There also exist a number of articles on 1-coverings from a purely geometric point of view, especially for generalized polygons; see [7, 8, 14].

2-coverings, on the other hand, are important when studying locally homogeneous graphs. A graph $\Gamma$ is called locally homogeneous if for any pair $\mathbf{x}, \mathbf{y}$ of vertices of $\Gamma$ the induced subgraphs $\Gamma(\mathbf{x})$ and $\Gamma(\mathbf{y})$ on the neighbors of $\mathbf{x}$, resp. $\mathbf{y}$, are isomorphic. It is clear from the definitions that any 2-cover of a locally homogeneous graph is again locally homogeneous with the same local structure. Therefore, when studying locally homogeneous graphs, it suffices to only consider the simply connected ones, i.e., those graphs that coincide with their universal 2-cover. For example, in [9] one can find a characterization of the line-hyperline graphs of a projective space of sufficiently large dimension by their local structure. The line-hyperline graph of some projective space $\mathbb{P}$ consists of the nonintersecting line-hyperline pairs of $\mathbb{P}$ (i.e., pairs of complemented subspaces of dimension two, resp. codimension two, in the corresponding vector space) in which the pair $(l, L)$ is adjacent to the pair $(m, M)$ if and only if $l \subseteq M$ and $m \subseteq L$. It is easily seen that the local structure of the line-hyperline graph of $\mathbb{P}$ is given by the line-hyperline graph of an arbitrary hyperline of $\mathbb{P}$. Conversely, Theorem 1 of [9] says that this local property is characteristic for the line-hyperline graphs for sufficiently large dimension. The proof of that theorem heavily relies on the assumption that the locally homogeneous graphs under consideration are simply connected. Only after a successful classification of the simply connected graphs does one realize that they do not admit quotients with the same local structure (because their diameter is two), completing the proof.

Surowski's work [18 beautifully unifies the concepts of 1-coverings and 2-coverings by considering simplicial complexes. While topologists may not be surprised by the results presented in [18, the article describes graph theoretic covering theory in a language perfectly suited for geometers.

Malnic, Nedela and Skoviera [13] define a different notion of graphs. A graph $\Gamma=(V, D, \iota,-1)$ in their sense consists of a set $V$ of vertices, a set $D$ of darts, a map $\iota: D \rightarrow V$ and a permutation $-1: D \rightarrow D: d \mapsto d^{-1}$ with $(-1)^{2}=$ id. The map $\iota$ assigns to each dart its initial vertex, while the map -1 interchanges a dart and its reverse. The terminal vertex of a dart $x$ is the initial vertex of $x^{-1}$. The orbits of -1 are called edges. Note that edges consist of one or two darts. An edge is called a semi-edge if its cardinality is one, a loop if its cardinality is two and both darts contained in this edge have the same initial vertex, and a link otherwise.

The authors of $[13$ justify this definition of a graph by applications in the theory of Cayley graphs. For example, Gross and Tucker [1] note that not all Cayley graphs are regular covers of bouquets of circles. Using the notion of a graph as in [13, however, one can prove that each Cayley graph is a regular cover of some 
monopole, i.e., a graph consisting of a unique vertex and an arbitrary number of darts, cf. 6.2 of [13], restated and reproved as Theorem 5.6 in the present paper. A covering $\phi$ in the sense of [13] is a graph surjection $\pi$ such that for each vertex $\mathbf{x}$ in the domain the set of darts with initial vertex $\mathbf{x}$ is mapped bijectively under $\pi$ onto the set of darts with initial vertex $\mathbf{x}^{\phi}$.

Neither topology nor Surowski's work [18] apply to the covering theory of graphs with semi-edges. Therefore it is one goal of the present paper to provide a suitable theory of coverings. To this end we define the notion of a fundamental 1-covering (see Definition 3.1) and prove that this covering is universal (cf. Definition 3.3).

Theorem 3.5. Let $\Gamma$ be a connected graph, let $\mathbf{x}$ be a vertex of $\Gamma$, and let $\widehat{\Gamma}$ be the fundamental 1-cover of $\Gamma$ based at $\mathbf{x}$. Then the fundamental 1-covering $\pi: \widehat{\Gamma} \rightarrow \Gamma$ is universal.

As a consequence of the preceding theorem we can classify all graphs that are 1-covers of a given graph. Results of this kind are typical in the context of topological spaces. In our context, however, we can achieve more: We give an explicit construction of each cover, comparable to the construction of covers of simplicial complexes in $\S 55$ of Seifert and Threlfall [15].

Later in this paper we turn our attention to reflections and local reflections of graphs, a reflection of a connected graph being an involutive automorphism of the graph that does not stabilize any vertex with the property that if one removes the darts of the graph that are normalized by the automorphism, then the graph becomes disconnected (see the beginning of Section 44). Applying the theory of reflections of graphs to the Cayley graph of a Coxeter group we obtain the following characterization.

Theorem 7.6. The following statements are equivalent:

(i) $(W, S)$ is a Coxeter system.

(ii) $(W, S)$ satisfies the exchange condition.

(iii) The elements of $S$ act as reflections on the Cayley graph Cay $(W, S)$.

In [12] this characterization is proven for the context of 'classical graphs' and used to identify as Coxeter groups reflection groups on certain topological spaces.

Finally, we combine the preceding theorem with our discussion of monopoles in Section 5 to obtain a characterization of the Cayley graphs of Coxeter groups.

Theorem 8.1. Let $\pi: \Gamma \rightarrow(v, D, \iota,-1)$ be a 1-covering of a monopole. The graph $\Gamma$ is the Cayley graph of a Coxeter group if and only if $\pi$ is regular and any deck transformation in $\Delta(\pi)$ that interchanges two neighboring vertices of $\Gamma$ acts as a reflection on $\Gamma$.

\section{Coverings}

Following 13, a graph $\Gamma=(V, D, \iota,-1)$ consists of a set $V$ of vertices, a set $D$ of darts, a map $\iota: D \rightarrow V$ and a permutation $-1: D \rightarrow D: d \mapsto d^{-1}$ with $(-1)^{2}=$ id. The map $\iota$ assigns to each dart its initial vertex, while the map -1 interchanges a dart and its reverse. The terminal vertex of a dart $x$ is the initial vertex of $x^{-1}$. The orbits of -1 are called edges. Note that edges consist of one or two darts. An edge is called a semi-edge if its cardinality is one, a loop if its cardinality is two and both darts contained in this edge have the same initial vertex, and a link otherwise. 
A monopole is a graph consisting of one vertex and a number of darts.

A morphism of graphs $\phi:\left(V_{1}, D_{1}, \iota_{1},-1_{1}\right) \rightarrow\left(V_{2}, D_{2}, \iota_{2},-1_{2}\right)$ consists of maps $V_{1} \rightarrow V_{2}$ and $D_{1} \rightarrow D_{2}$ such that $\iota_{1} \phi=\phi \iota_{2}$ and $\left(-1_{1}\right) \phi=\phi\left(-1_{2}\right)$.

A path of length $t$ is a sequence of $t$ darts $\gamma=x_{1} \cdots x_{t}$ such that the terminal vertex of $x_{k}$ coincides with the initial vertex of $x_{k+1}$ for all $1 \leq k \leq t-1$. A path of length 0 (or trivial path) is a vertex $\mathbf{x}$. The initial vertex of $\gamma$, denoted by $\gamma^{\iota}$, is the initial vertex of $x_{1}$ (resp. $\mathbf{x}$ in the case of a trivial path), the terminal vertex of $\gamma$ is the terminal vertex of $x_{t}$ (resp. $\mathbf{x}$ in the case of a trivial path). If a path $\gamma$ has initial vertex $\mathbf{x}$, then $\gamma$ is based at $\mathbf{x}$. If its initial and terminal vertices coincide, then $\gamma$ is called a cycle or a closed path.

If a path $\gamma_{1}$ terminates at some vertex $\mathbf{x}$ and a path $\gamma_{2}$ starts at the same vertex $\mathbf{x}$, then $\gamma_{1} \gamma_{2}$ is a path, called the concatenation of $\gamma_{1}$ and $\gamma_{2}$. Note that concatenation with the trivial path does not change the path. Furthermore, $\left(\gamma_{1} \gamma_{2}\right)^{\iota}=\gamma_{1}^{\iota}$ and $\left(\gamma_{1} \gamma_{2}\right)^{-1}=\gamma_{2}^{-1} \gamma_{1}^{-1}$.

A graph is connected if for each pair $\mathbf{x}, \mathbf{y}$ there exists a path from $\mathbf{x}$ to $\mathbf{y}$. We say that a vertex $\mathbf{y}$ is a neighbor of the vertex $\mathbf{x}$ if there exists a dart $d$ with $d^{\iota}=\mathbf{x}$ and $\left(d^{-1}\right)^{\iota}=\mathbf{y}$. Notice that the neighbor relation is symmetric. We denote the set of all neighbors of some vertex $\mathbf{x}$ by $B_{1}(\mathbf{x})$ (' $\mathrm{B}$ ' stands for ball) and the set $B_{1}(\mathbf{x}) \cup\{\mathbf{x}\}$ by $D_{1}(\mathbf{x})$ ('D' stands for disk).

Let $\Gamma$ be a connected graph. Two paths in $\Gamma$ are 1-homotopic if one can be obtained from the other by a finite number of applications of the following operation, called elementary 1-homotopy: inserting or deleting a return, i.e., a cycle $x x^{-1}$ of length two: replace $x x^{-1}$ by $x^{\iota}$ or $x^{\iota}$ by $x x^{-1}$. A cycle that is homotopic to a cycle of length 0 is called 1-homotopically trivial. By $\amalg_{1}(\Gamma)$ denote the 1-fundamental groupoid (also called path groupoid) of $\Gamma$, that is, the set of all 1-homotopy classes of paths in $\Gamma$ endowed with the partial multiplication $\left(\left[\gamma_{1}\right]_{1},\left[\gamma_{2}\right]_{1}\right) \mapsto\left[\gamma_{1} \gamma_{2}\right]_{1}$ whenever the terminal vertex of $\gamma_{1}$ coincides with the initial vertex of $\gamma_{2}$; the subset $\amalg_{1}(\Gamma, \mathbf{x}) \subset \amalg_{1}(\Gamma)$ of 1-homotopy classes of paths in $\Gamma$ with initial vertex $\mathbf{x}$ is called the path groupoid of $\Gamma$ based at $\mathbf{x}$. The subset $\Pi_{1}(\Gamma, \mathbf{x}) \subset \amalg_{1}(\Gamma, \mathbf{x}) \subset \amalg_{1}(\Gamma)$ of all 1-homotopy classes of cycles in $\Gamma$ based at some vertex $\mathbf{x}$ forms a group, the 1-fundamental group (or path group) of $\Gamma$ at $\mathrm{x}$.

Lemma 2.1. Let $\Gamma$ be a connected graph and let $\mathbf{x}, \mathbf{y}$ be vertices of $\Gamma$. Then $\Pi_{1}(\Gamma, \mathbf{x}) \cong \Pi_{1}(\Gamma, \mathbf{y})$ via the isomorphism that, for a fixed path $\gamma$ from $\mathbf{y}$ to $\mathbf{x}$, assigns to a homotopy class $[\delta]_{1}$ of cycles based at $\mathbf{x}$ the homotopy class $\left[\gamma \delta \gamma^{-1}\right]_{1}$ of cycles based at $\mathbf{y}$.

Proof. As $\left[\gamma \delta_{1} \gamma^{-1}\right]_{1}\left[\gamma \delta_{2} \gamma^{-1}\right]_{1}=\left[\gamma \delta_{1} \gamma^{-1} \gamma \delta_{2} \gamma^{-1}\right]_{1}=\left[\gamma \delta_{1} \delta_{2} \gamma^{-1}\right]_{1}$ the given map is a group homomorphism. Certainly, it is bijective, so it is an isomorphism.

In a connected graph $\Gamma$, by the above lemma, two path groups with base points $\mathbf{x}, \mathbf{y}$ are isomorphic under the transition map that, for a fixed path $\gamma$ from $\mathbf{y}$ to $\mathbf{x}$, assigns to a homotopy class $[\delta]_{1}$ of paths based at $\mathbf{x}$ the homotopy class $\left[\gamma \delta \gamma^{-1}\right]_{1}$ of paths based at $\mathbf{y}$. Any representative of this isomorphism class of path groups of $\Gamma$ with base point is called the path group of $\Gamma$, denoted by $\Pi_{1}(\Gamma)$. The corresponding transition map is called a base transformation.

Definition 2.2. Let $\Gamma=(V, D, \iota,-1)$ and $\widehat{\Gamma}=(\widehat{V}, \widehat{D}, \widehat{\iota}, \widehat{-1})$ be graphs. A graph epimorphism $\pi: \widehat{\Gamma} \rightarrow \Gamma$ is called a 1-covering if, for every vertex $\mathbf{x} \in \widehat{\Gamma}$, the map $\pi$ sends the set of darts of $\widehat{\Gamma}$ with initial vertex $\mathbf{x}$ bijectively onto the set of darts 
of $\Gamma$ with initial vertex $\mathbf{x}^{\pi}$. The graph $\widehat{\Gamma}$ is called a 1-cover of the graph $\Gamma$. The set $\pi^{-1}(x), x \in V \cup D$ is called a fiber; it is called a vertex fiber if $x \in V$ and a dart fiber if $x \in D$.

A connected graph $\Gamma$ is called 1-simply connected if any 1-covering $\widehat{\Gamma} \rightarrow \Gamma$ with $\widehat{\Gamma}$ connected is an isomorphism.

Lemma 2.3. Let $\pi: \widehat{\Gamma} \rightarrow \Gamma$ be a 1-covering of graphs and let $\gamma$ be an arbitrary path in $\Gamma$ with initial vertex $\mathbf{x}$. Then for every vertex $\widehat{\mathbf{x}} \in \pi^{-1}(\mathbf{x})$ there exists a unique path $\widehat{\gamma}$ with $\widehat{\mathbf{x}}$ as initial vertex and $\widehat{\gamma}^{\pi}=\gamma$. Moreover, 1-homotopic paths of $\Gamma$ lift to 1-homotopic paths of $\widehat{\Gamma}$, and 1-homotopic paths of $\widehat{\Gamma}$ are mapped onto 1-homotopic paths of $\Gamma$. In particular, if $\gamma_{1}$ and $\gamma_{2}$ are 1-homotopic in $\Gamma$, then their lifts $\widehat{\gamma}_{1}$ and $\widehat{\gamma}_{2}$ in $\widehat{\Gamma}$ with identical initial vertices have identical terminal vertices.

Proof. See Proposition 4.2 of [13].

Let $\pi: \widehat{\Gamma} \rightarrow \Gamma$ be a 1-covering of graphs and let $\phi$ be an automorphism of $\Gamma$. We say that $\phi$ lifts to $\widehat{\Gamma}$ if there exists an automorphism $\widehat{\phi}$ of $\widehat{\Gamma}$, a lift of $\phi$, such that $\hat{\phi} \pi=\pi \phi$. If $\phi$ lifts, then so does $\phi^{-1}$. If, more generally, all automorphisms in a group $A \leq$ Aut $\Gamma$ lift, then all those lifts form a group, the lift $\widehat{A} \leq$ Aut $\widehat{\Gamma}$ of $A$. (See [13] for conditions under which lifts exist.) The trivial group of automorphisms of $\Gamma$ always lifts. The group of all of its lifts is called the group of deck transformations and is denoted by $\Delta(\pi)$.

Proposition 2.4. Let $\pi: \widehat{\Gamma} \rightarrow \Gamma$ be a 1-covering of connected graphs. Then $\Delta(\pi)$ acts semi-regularly on $\widehat{\Gamma}$; that is, $\Delta(\pi)$ acts fixed point-freely on both the set of vertices and the set of darts of $\widehat{\Gamma}$.

Proof. This follows directly from Lemma 2.3 .

Definition 2.5. Let $\pi: \widehat{\Gamma} \rightarrow \Gamma$ be a 1-covering of connected graphs. It is called regular if $\Delta(\pi)$ acts transitively on some, whence each, vertex fiber.

The implication of transitivity on each vertex fiber by the transitivity on some vertex fiber follows from Lemma 2.3 as follows. First, one observes that by the connectedness of $\Gamma$, it is enough to show that if $\Delta(\pi)$ acts transitively on $\pi^{-1}(\mathbf{x})$ for some vertex $\mathbf{x}$ of $\Gamma$ it acts transitively on $\pi^{-1}(\mathbf{y})$ for all neighbors $\mathbf{y}$ of $\mathbf{x}$. So, let $d$ be a dart with $d^{\iota}=\mathbf{x}$ and $\left(d^{-1}\right)^{\iota}=\mathbf{y}$. Then by Lemma 2.3, the path $d$ lifts to a unique path $\widehat{d}$ for each $\widehat{\mathbf{x}} \in \pi^{-1}(\mathbf{x})$. Hence, if $\delta \in \Delta(\pi)$ maps $\widehat{\mathbf{x}}_{1}$ onto $\widehat{\mathbf{x}}_{2}$, it also maps the corresponding lift $\widehat{d}_{1}$ of $d$ starting at $\widehat{\mathbf{x}}_{1}$ onto the lift $\widehat{d}_{2}$ of $d$ starting at $\widehat{\mathbf{x}}_{1}$, whence $\left(\widehat{d}_{1}^{-1}\right)^{\iota}$ gets mapped onto $\left(\widehat{d}_{2}^{-1}\right)^{\iota}$. The transitivity of $\Delta(\pi)$ on the fiber of $\pi^{-1}(\mathbf{y})$ now follows from the fact that each element of the fiber of $\mathbf{y}$ is a neighbor of an element of the fiber of $\mathbf{x}$.

Remark 2.6. It seems tempting to extend the theory of 2-coverings to graphs admitting loops and semi-edges as well. However, this does not lead to any new concept at all. For, when studying 2-coverings of graphs one requires that cycles of the form $d_{1} d_{2} d_{3}$ with $d_{1}^{\iota}=\left(d_{3}^{-1}\right)^{\iota}$ are null-homotopic. For a semi-edge $d$ this implies that $d$ is null-homotopic, because both $d d$ and $d d d$ are null-homotopic. Similarly, if $l$ is a loop based at the vertex $\mathbf{x}$ and $d$ is any other dart based at $\mathbf{x}$, then $l$ is null-homotopic because $l d d^{-1}$ and $d d^{-1}$ are null-homotopic. Therefore the only connected graph in which loops and semi-edges do not lift trivially is the graph 
consisting of one vertex and a loop, whose universal 2-cover is the complete graph on three vertices.

\section{Fundamental AND Universal COVERS}

Definition 3.1. Let $\Gamma=(V, D, \iota,-1)$ be a connected graph and let $\mathbf{x}$ be a vertex of $\Gamma$. Then the graph $\widehat{\Gamma}=(\widehat{V}, \widehat{D}, \widehat{\iota}, \widehat{-1})$ with

$$
\begin{aligned}
\widehat{V} & =\amalg_{1}(\Gamma, \mathbf{x}), \\
\widehat{D} & =\left\{\left([\gamma]_{1}, d\right) \in \amalg_{1}(\Gamma, \mathbf{x}) \times D \mid d^{\iota}=\left(\gamma^{-1}\right)^{\iota}\right\}, \\
\widehat{\iota}: \widehat{D} \rightarrow \widehat{V} & : \quad\left([\gamma]_{1}, d\right) \mapsto[\gamma]_{1}, \\
\widehat{-1}: \widehat{D} \rightarrow \widehat{D} & : \quad\left([\gamma]_{1}, d\right) \mapsto\left([\gamma d]_{1}, d^{-1}\right)
\end{aligned}
$$

is called the fundamental 1-cover of $\Gamma$ based at $\mathbf{x}$.

Proposition 3.2. Let $\Gamma$ be a connected graph, let $\mathbf{x}$ be a vertex of $\Gamma$, and let $\widehat{\Gamma}$ be the fundamental 1-cover of $\Gamma$ based at $\mathbf{x}$. Then the canonical map $\pi: \widehat{\Gamma} \rightarrow \Gamma$ with

$$
\begin{array}{ll}
\left.\pi\right|_{\widehat{V}}: \widehat{V} \rightarrow V & : \quad \gamma \mapsto\left(\gamma^{-1}\right)^{\iota}, \\
\left.\pi\right|_{\widehat{D}}: \widehat{D} \rightarrow D & : \quad\left([\gamma]_{1}, d\right) \mapsto d
\end{array}
$$

is a 1-covering map. Moreover, $\pi: \widehat{\Gamma} \rightarrow \Gamma$ is an isomorphism if and only if $\Pi_{1}(\Gamma, \mathbf{x})$ is trivial.

Proof. Straightforward.

Definition 3.3. Let $\Gamma, \widehat{\Gamma}$ be connected graphs and let $\mathbf{x} \in \Gamma, \widehat{\mathbf{x}} \in \widehat{\Gamma}$ be vertices. A 1-covering $\pi: \widehat{\Gamma} \rightarrow \Gamma$ mapping $\widehat{\mathbf{x}}$ onto $\mathbf{x}$ is called universal if, for any 1-covering $\alpha: \Gamma_{1} \rightarrow \Gamma$ and any $\mathbf{x}_{1} \in \alpha^{-1}(\mathbf{x})$, there exists a unique 1-covering map $\beta: \widehat{\Gamma} \rightarrow \Gamma_{1}$ with $\pi=\beta \alpha$ and $\widehat{\mathbf{x}}^{\beta}=\mathbf{x}_{1}$.

Remark 3.4. By definition a universal 1-covering $\pi: \widehat{\Gamma} \rightarrow \Gamma$ is regular. Indeed, if $\mathbf{x} \in \Gamma, \widehat{\mathbf{x}} \in \widehat{\Gamma}$ with $\widehat{\mathbf{x}}^{\pi}=\mathbf{x}$, then, by the universality of $\pi$, for each $\mathbf{x}_{1} \in \pi^{-1}(\mathbf{x})$ there exists an automorphism of $\widehat{\Gamma}$ mapping $\widehat{\mathbf{x}}$ onto $\mathbf{x}_{1}$.

Theorem 3.5. Let $\Gamma$ be a connected graph, let $\mathbf{x}$ be a vertex of $\Gamma$, and let $\widehat{\Gamma}$ be the fundamental 1-cover of $\Gamma$ based at $\mathbf{x}$. Then the fundamental 1-covering $\pi: \widehat{\Gamma} \rightarrow \Gamma$ is universal.

Proof. Let $\alpha: \Gamma_{1} \rightarrow \Gamma$ be an arbitrary 1-covering and let $\mathbf{x}_{1} \in \alpha^{-1}(\mathbf{x})$. By Lemma 2.3 any class of paths $[\gamma]_{1} \in \amalg_{1}(\Gamma, \mathbf{x})$ lifts to a unique class of paths $[\widehat{\gamma}]_{1} \in \amalg_{1}\left(\Gamma_{1}, \mathbf{x}_{1}\right)$. Define a map $\beta: \widehat{\Gamma} \rightarrow \Gamma_{1}$ by $[\gamma]_{1}^{\beta}=\left(\widehat{\gamma}^{-1}\right)^{\iota}$ and $\left([\gamma]_{1}, d\right)^{\beta}=\widehat{d}$, where $\widehat{d}$ is the unique lift of $d$ based at $[\gamma]_{1}^{\beta}=\left(\widehat{\gamma}^{-1}\right)^{\iota}$ by Lemma 2.3. It is clear that $\beta: \widehat{\Gamma} \rightarrow \Gamma_{1}$ is a 1-covering with $\pi=\beta \alpha$ and $\mathbf{x}^{\beta}=\mathbf{x}_{1}$. Uniqueness follows from connectedness.

Corollary 3.6. Let $\Gamma$ be a connected graph and let $\mathbf{x}$ be a vertex of $\Gamma$. Then its fundamental 1-cover $\widehat{\Gamma}$ based at $\mathbf{x}$ is 1-simply connected.

Proof. Let $\pi: \widehat{\Gamma} \rightarrow \Gamma$ be the fundamental 1-covering and let $\phi: \Gamma_{1} \rightarrow \widehat{\Gamma}$ be some 1-covering. Then $\alpha:=\phi \pi: \Gamma_{1} \rightarrow \Gamma$ is a 1-covering. Let $\mathbf{x}_{1} \in \phi^{-1}(\mathbf{x}) \subseteq \alpha^{-1}(\mathbf{x})$. By the universal property of $\pi$, there exists a 1-covering $\beta: \widehat{\Gamma} \rightarrow \Gamma_{1}$ mapping (x) onto $\mathbf{x}_{1}$ with $\pi=\beta \alpha=\beta \phi \pi$. Then $\beta \phi$ is a 1-covering from $\widehat{\Gamma}$ onto itself with 
$(\mathbf{x})^{\beta \phi}=(\mathbf{x})$, whence it is the identity by the universal property of $\pi$. Therefore $\beta$ is injective, whence bijective. Thus the graph morphism $\phi$ is the inverse of the graph morphism $\beta$ and both $\beta$ and $\phi$ are graph automorphisms. Hence $\widehat{\Gamma}$ is 1 -simply connected.

Let $\Gamma$ be a connected graph. If $\widehat{\Gamma}_{1}$ is the fundamental 1-cover of $\Gamma$ based at $\mathbf{x}$ and $\widehat{\Gamma}_{2}$ is the fundamental 1-cover of $\Gamma$ based at $\mathbf{y}$, and, moreover, if $\gamma$ is an arbitrary path in $\Gamma$ from $\mathbf{y}$ to $\mathbf{x}$, then the map from $\widehat{\Gamma}_{1}$ to $\widehat{\Gamma}_{2}$ induced by $[\delta]_{1} \mapsto[\gamma \delta]_{1}$ is an isomorphism of graphs. This proves the following.

Proposition 3.7. Let $\Gamma$ be a connected graph and let $\mathbf{x}, \mathbf{y}$ be vertices of $\Gamma$. Then the fundamental 1-cover of $\Gamma$ based at $\mathbf{x}$ is isomorphic to the fundamental 1-cover of $\Gamma$ at $\mathbf{y}$ via base transformation.

Hence we can suppress the base point of a fundamental 1-cover and speak of the fundamental 1-cover of $\Gamma$. If we speak of a fundamental 1-cover and a path group in the same context, then we always assume that the particular cover and the particular group have the same base vertex.

Corollary 3.8 (of Theorem 3.5). Let $\Gamma$ be a connected graph. The graph $\Gamma$ is 1-simply connected if and only if $\Pi_{1}(\Gamma)$ is trivial.

Proof. If $\Pi_{1}(\Gamma)$ is non-trivial, then the fundamental cover of $\Gamma$ is not isomorphic to $\Gamma$ by Proposition 3.2. Hence $\Gamma$ is not simply connected.

Conversely, assume that $\Gamma$ is not simply connected. Then it admits a 1-covering $\alpha: \Gamma_{1} \rightarrow \Gamma$ for some $\Gamma_{1}$ that is not an isomorphism. If $\pi: \widehat{\Gamma} \rightarrow \Gamma$ is the canonical 1-covering of $\Gamma$ by its fundamental 1-cover $\widehat{\Gamma}$, then, by Theorem 3.5. there exists a 1-covering $\beta: \widehat{\Gamma} \rightarrow \Gamma_{1}$ with $\pi=\beta \alpha$. If $\pi$ were to be an isomorphism, then $\alpha$ would have to be injective (as $\beta$ is surjective), which is not the case. So $\pi$ is not an isomorphism and Proposition 3.2 implies that $\Pi_{1}(\Gamma)$ is non-trivial.

Corollary 3.9 (of Corollary 3.8). Let $\Gamma$ be a connected graph and let $\alpha: \Gamma_{1} \rightarrow \Gamma$ be a 1-covering with 1-simply connected $\Gamma_{1}$. Then $\Gamma_{1}$ is isomorphic to the fundamental 1-cover $\widehat{\Gamma}$ of $\Gamma$.

Theorem 3.10. Let $\Gamma=(V, D, \iota,-1)$ be a connected graph. Then, for any vertex $\mathbf{x}$ of $\Gamma$, there is a one-to-one correspondence between 1-covers of $\Gamma$ based at $\mathbf{x}$ and subgroups of $\Pi_{1}(\Gamma, \mathbf{x})$. More precisely, if $U$ is a subgroup of $\Pi_{1}(\Gamma, \mathbf{x})$, then the corresponding 1-cover of $\Gamma$ is the graph $\Gamma_{0}=\left(V_{0}, D_{0}, \iota_{0},-1_{0}\right)$ with

$$
\begin{aligned}
V_{0} & =U \backslash \amalg_{1}(\Gamma, \mathbf{x}), \\
D_{0} & =\left\{\left(U[\gamma]_{1}, d\right) \in U \backslash \amalg_{1}(\Gamma, \mathbf{x}) \times D \mid d^{\iota}=\left(\gamma^{-1}\right)^{\iota}\right\}, \\
\widehat{\iota}: D_{0} \rightarrow V_{0} & :\left(U[\gamma]_{1}, d\right) \mapsto U[\gamma]_{1}, \\
-1_{0}: D_{0} \rightarrow D_{0} & :\left(U[\gamma]_{1}, d\right) \mapsto\left(U[\gamma d]_{1}, d^{-1}\right) .
\end{aligned}
$$

Note that we use the symbol $\backslash$ in the theorem to denote right cosets, respectively $U$-orbits, for the left multiplication action on $\amalg_{1}(\Gamma, \mathbf{x})$.

Proof. Given a subgroup $U$ of $\Pi_{1}(\Gamma, \mathbf{x})$ it is straightforward to check that the graph $\Gamma_{0}$ given above is a 1 -cover of $\Gamma$.

Conversely, let $\alpha: \Gamma_{0} \rightarrow \Gamma$ be a 1-covering. By Theorem 3.5 there exists a 1-covering $\beta: \widehat{\Gamma} \rightarrow \Gamma_{0}$, where $\widehat{\Gamma}$ is the fundamental cover of $\Gamma$. We can consider 
$\widehat{\Gamma}$ as the fundamental 1-cover of $\Gamma_{0}$ based at $\mathbf{x}^{\beta}$, since $\widehat{\Gamma}$ is 1 -simply connected, so by Corollary 3.9 isomorphic to the fundamental 1-cover of $\Gamma_{0}$. By Lemma 2.3 the 1-covering $\alpha$ induces an embedding of $\Pi_{1}\left(\Gamma_{0}, \mathbf{x}^{\beta}\right)$ in $\Pi_{1}(\Gamma, \mathbf{x})$. (It induces a welldefined map as images of homotopic paths are homotopic. This induced map is injective as lifts of homotopic paths are homotopic.) We have identified $\Pi_{1}\left(\Gamma_{0}, \mathbf{x}^{\beta}\right)$ with a subgroup of $\Pi_{1}(\Gamma, \mathbf{x})$ and hence $\Gamma_{0}=\Pi_{1}\left(\Gamma_{0}, \mathbf{x}^{\beta}\right) \backslash \widehat{\Gamma}$, finishing the proof.

Corollary 3.11 (of Lemma 2.1 and Theorem 3.10). Let $\Gamma$ be a connected graph. Then there is a one-to-one correspondence between equivalence classes of 1-covers of $\Gamma$ without base vertex and conjugacy classes of subgroups of $\Pi_{1}(\Gamma)$.

Corollary 3.12 (of Theorem 3.10). Let $\pi: \Gamma_{1} \rightarrow \Gamma_{2}$ be a 1-covering and let $\mathbf{y}=\mathbf{x}^{\pi}$ for some vertex $\mathbf{x}$ of $\Gamma_{1}$. Then $\pi$ induces a group monomorphism $\pi_{\#}: \Pi\left(\Gamma_{1}, \mathbf{x}\right) \rightarrow$ $\Pi\left(\Gamma_{2}, \mathbf{y}\right)$.

Corollary 3.13 (of Theorem 3.10). Let $\pi: \Gamma_{1} \rightarrow \Gamma_{2}$ be a 1-covering with $\mathbf{y}=\mathbf{x}^{\pi}$ for some vertex $\mathbf{x} \in \Gamma_{1}$. Assume that $\alpha: \Gamma_{0} \rightarrow \Gamma_{2}$ is another 1-covering, and let $\mathbf{w} \in \alpha^{-1}(\mathbf{y})$. Then a 1-covering $\beta: \Gamma_{0} \rightarrow \Gamma_{1}$ with $\beta \pi=\alpha$ exists if and only if $\Pi_{1}\left(\Gamma_{0}, \mathbf{w}\right)^{\alpha \#} \subseteq \Pi_{1}\left(\Gamma_{1}, \mathbf{x}\right)^{\pi_{\#}}$. Moreover, if such a $\beta$ exists, it is unique.

Corollary $\mathbf{3 . 1 4}$ (of Corollary 3.13). Let $\pi: \Gamma_{1} \rightarrow \Gamma_{2}$ be a 1-covering with $\mathbf{y}=\mathbf{x}^{\pi}$ for some vertex $\mathbf{x} \in \Gamma_{1}$ and let $\mathbf{x}_{0} \in \pi^{-1}(\mathbf{x})$. Then there exists a deck transformation in $\Delta(\pi)$ taking $\mathbf{x}_{0}$ to $\mathbf{x}$ if and only if $\Pi_{1}\left(\Gamma_{1}, \mathbf{x}_{0}\right)^{\pi_{\#}}=\Pi_{1}\left(\Gamma_{1}, \mathbf{x}\right)^{\pi_{\#}}$.

Proof. Let $\phi \in \Delta(\pi)$ be the deck transformation taking $\mathbf{x}_{0}$ to $\mathbf{x}$. Then Corollary 3.13 applies with $\Gamma_{0}=\Gamma_{1}, \mathbf{w}=\mathbf{x}_{0}, \beta=\phi$ and $\alpha=\phi \pi=\pi$, yielding $\Pi_{1}\left(\Gamma_{1}, \mathbf{x}_{0}\right)^{(\phi \pi)_{\#}}=\Pi_{1}\left(\Gamma_{1}, \mathbf{x}_{0}\right)^{\pi_{\#}} \subseteq \Pi_{1}\left(\Gamma_{1}, \mathbf{x}\right)^{\pi_{\#}}$. The reverse inclusion follows by symmetry.

Corollary 3.15 (of Lemma 2.1 and Corollary 3.14 cf. Corollary 5.5 of [13]). Let $\pi: \Gamma_{1} \rightarrow \Gamma_{2}$ be a 1-covering with $\mathbf{y}=\mathbf{x}^{\pi}$ for some vertex $\mathbf{x} \in \Gamma_{1}$. The subgroup $\Pi_{1}\left(\Gamma_{1}, \mathbf{x}\right)^{\pi_{\#}}$ of $\Pi_{1}\left(\Gamma_{2}, \mathbf{y}\right)$ is normal if and only if $\pi$ is a regular 1-covering.

Proof. The base transformation of Lemma 2.1 between bases $\mathbf{x}_{0}, \mathbf{x}$ inside the fiber $\pi^{-1}(\mathbf{y})$ proves that $\Pi_{1}\left(\Gamma_{1}, \mathbf{x}\right)^{\pi_{\#}}$ and $\Pi_{1}\left(\Gamma_{1}, \mathbf{x}_{0}\right)^{\pi_{\#}}$ are conjugate inside $\Pi_{1}\left(\Gamma_{2}, \mathbf{y}\right)$. On the other hand, by Corollary 3.14, we have $\Pi_{1}\left(\Gamma_{1}, \mathbf{x}_{0}\right)^{\pi_{\#}}=\Pi_{1}\left(\Gamma_{1}, \mathbf{x}\right)^{\pi_{\#}}$ for all $\mathbf{x}_{0} \in \pi^{-1}(\mathbf{y})$ if and only if $\Delta(\pi)$ acts transitively on $\pi^{-1}(\mathbf{y})$, i.e., if and only if $\pi$ is a regular 1-covering.

Corollary 3.16 (of Corollary 3.15). Let $\pi: \Gamma_{1} \rightarrow \Gamma_{2}$ be a regular 1-covering with $\mathbf{y}=\mathbf{x}^{\pi}$ for some vertex $\mathbf{x} \in \Gamma_{1}$. Then

$$
\Delta(\pi) \cong \Pi_{1}\left(\Gamma_{2}, \mathbf{y}\right) / \Pi_{1}\left(\Gamma_{1}, \mathbf{x}\right)^{\pi_{\#}} .
$$

Corollary 3.17 (of Corollaries 3.8 and 3.16). Let $\pi: \Gamma_{1} \rightarrow \Gamma_{2}$ be a regular 1covering with $\mathbf{y}=\mathbf{x}^{\pi}$ for some vertex $\mathbf{x} \in \Gamma_{1}$. If, moreover, $\Gamma_{1}$ is 1-simply connected, then

$$
\Delta(\pi) \cong \Pi_{1}\left(\Gamma_{2}, \mathbf{y}\right)
$$

Remark 3.18. The concept of a fundamental cover for simplicial complexes already exists in the literature, e.g. $\S 55$ of Seifert and Threlfall [15. The purpose of Section 3 is to provide an extension of the simplicial covering theory to graphs with semiedges. 


\section{Reflections AND LOCAL REFLECTIONS}

For an automorphism $\sigma$ of a connected graph $\Gamma=(V, D, \iota,-1)$ set

$\operatorname{Fix}_{\sigma}(V):=\left\{v \in V \mid v^{\sigma}=v\right\}$, the set of fixed vertices, and $\operatorname{Norm}_{\sigma}(D):=\left\{d \in D \mid d \neq d^{\sigma}=d^{-1}\right\}$, the set of properly normalized darts.

An involution $\sigma$ of a connected graph $\Gamma=(V, D, \iota,-1)$ is called a reflection if $\operatorname{Fix}_{\sigma}(V)=\emptyset$, and if $\Gamma_{\sigma}=\left(V, D_{\sigma}, \iota_{\sigma},-1_{\sigma}\right)$ with $D_{\sigma}=D \backslash \operatorname{Norm}_{\sigma}(D)$ and $\iota_{\sigma}=\iota_{D_{\sigma}}$, $-1_{\sigma}=-\left.1\right|_{D_{\sigma}}$ is disconnected. We write $x \sim_{\sigma} x^{\prime}$ if $x$ and $x^{\prime}$ are vertices of the same connected component in $\Gamma_{\sigma}$ and we say that $\sigma$ separates $x$ and $x^{\prime}$ if we have $x \varkappa_{\sigma} x^{\prime}$. For $x \in V$ set

$$
V_{+}^{\sigma}(x)=\left\{y \in V \mid x \sim_{\sigma} y\right\} \quad \text { and } \quad V_{-}^{\sigma}(x)=\left\{y \in V \mid x \varkappa_{\sigma} y\right\} .
$$

An involution $\sigma$ of a connected graph $\Gamma$ is called a local reflection if there exists a vertex $v$ with $D_{1}(v) \cap D_{1}\left(v^{\sigma}\right) \neq \emptyset$ and if, for any vertex $v$ with $D_{1}(v) \cap D_{1}\left(v^{\sigma}\right) \neq \emptyset$, the restriction of $\sigma$ to $D_{1}(v) \cap D_{1}\left(v^{\sigma}\right)$ is a reflection.

Proposition 4.1. Let $\Gamma=(V, D, \iota,-1)$ be a connected graph. Any local reflection $\sigma: \Gamma \rightarrow \Gamma$ has the following properties. The set $\operatorname{Fix}_{\sigma}(V)$ is empty, the graph $\Gamma_{\sigma}$ consists of one or two connected components, and, for every dart $d$ with $d \neq d^{\sigma}=$ $d^{-1}$, the graph $\left(V, D_{\sigma} \cup\left\{d, d^{-1}\right\},\left.\iota\right|_{D_{\sigma} \cup\left\{d, d^{-1}\right\}},-\left.1\right|_{D_{\sigma} \cup\left\{d, d^{-1}\right\}}\right)$ is connected.

Proof. Suppose $v \in \operatorname{Fix}_{\sigma}(V)$. Then $D_{1}(v) \cap D_{1}\left(v^{\sigma}\right)$ contains $v=v^{\sigma}$, so the restriction of $\sigma$ to $D_{1}(v) \cap D_{1}\left(v^{\sigma}\right)$ is not a reflection.

Suppose $\Gamma_{\sigma}$ consists of more than two connected components. Since darts of $\Gamma$ with $d \neq d^{\sigma}=d^{-1}$ can only connect connected components of $\Gamma_{\sigma}$ that are interchanged by $\sigma$, the original graph $\Gamma$ cannot be connected, a contradiction. Hence $\Gamma_{\sigma}$ consists of one or two connected components.

The last statement is trivially true if $\Gamma_{\sigma}$ is connected. If it is disconnected, then, by the above, it consists of two connected components, and the connected components have to be interchanged by $\sigma$. Adding any dart of $\Gamma$ with $d \neq d^{\sigma}=d^{-1}$ obviously connects the two components.

Corollary 4.2. Let $\Gamma=(V, D, \iota,-1)$ be a connected graph. A local reflection of $\Gamma$ is a reflection if and only if $\Gamma_{\sigma}$ consists of two connected components.

Theorem 4.3. Let $\Gamma=(V, D, \iota,-1)$ be a connected graph. Any local reflection $\sigma$ of $\Gamma$ can be lifted to a reflection $\widehat{\sigma}$ of $\widehat{\Gamma}=(\widehat{V}, \widehat{D}, \widehat{\iota}, \widehat{-1})$ with

$$
\begin{aligned}
\widehat{V} & =V \times\{-1,1\}, \\
\widehat{D} & =D \times\{-1,1\}, \\
\widehat{\iota}: \widehat{D} \rightarrow \widehat{V} & : \quad(d, \pm 1) \mapsto\left(d^{\iota}, \pm 1\right) \\
\widehat{-1}: \widehat{D} \rightarrow \widehat{D} & : \quad(d, \pm 1) \mapsto \begin{cases}\left(d^{-1}, \pm 1\right), & \text { if } d \notin \operatorname{Norm}_{\sigma}(D), \\
\left(d^{-1}, \mp 1\right), & \text { if } d \in \operatorname{Norm}_{\sigma}(D),\end{cases}
\end{aligned}
$$

mapping $(v, \pm 1)$ onto $\left(v^{\sigma}, \mp 1\right)$ and $(d, \pm 1)$ onto $\left(d^{\sigma}, \mp 1\right)$. The canonical map $\pi$ : $\widehat{\Gamma} \rightarrow \Gamma$ is a 1 -covering and $\sigma \pi=\pi \widehat{\sigma}$. 
Proof. The only thing to prove is the fact that $\widehat{\sigma}$ is a reflection. It is a graph morphism, since

$$
\begin{aligned}
\left((d, \pm 1)^{\iota}\right)^{\widehat{\sigma}} & =\left(d^{\iota}, \pm 1\right)^{\widehat{\sigma}} \\
& =\left(d^{\iota \sigma}, \mp 1\right) \\
& =\left(d^{\sigma \iota}, \mp 1\right) \\
& =\left(d^{\sigma}, \mp 1\right)^{\iota} \\
& =\left((d, \pm 1)^{\widehat{\sigma}}\right)^{\iota}
\end{aligned}
$$

and

$$
\begin{aligned}
\left((d, \pm 1)^{-1}\right)^{\widehat{\sigma}} & =\left(d^{-1}, \epsilon( \pm 1)\right)^{\widehat{\sigma}} \\
& =\left(d^{(-1) \sigma}, \epsilon(\mp 1)\right) \\
& =\left(d^{\sigma(-1)}, \epsilon(\mp 1)\right) \\
& =\left(d^{\sigma}, \mp 1\right)^{-1} \\
& =\left((d, \pm 1)^{\widehat{\sigma}}\right)^{-1},
\end{aligned}
$$

where $\epsilon=1$ if $d \notin \operatorname{Norm}_{\sigma}(D)$ and $\epsilon=-1$ if $d \in \operatorname{Norm}_{\sigma}(D)$. Because $\widehat{\sigma}^{2}=$ id the morphism $\widehat{\sigma}$ is an automorphism. Certainly, the graph $\widehat{\Gamma}$ is connected and $\operatorname{Fix}_{\widehat{\sigma}}(\widehat{V})=\emptyset$. The set $\operatorname{Norm}_{\widehat{\sigma}}(\widehat{D})$ equals $\left\{(d, \pm 1) \mid d \in \operatorname{Norm}_{\sigma}(D)\right\}$ and the graph $\widehat{\Gamma}_{\widehat{\sigma}}$ consists of two connected components. Therefore $\widehat{\sigma}$ is a reflection.

Lemma 4.4. If $\sigma$ is a (local) reflection on a graph $\Gamma=(V, D, \iota,-1)$ and $\phi$ is a graph automorphism of $\Gamma$, then $\phi^{-1} \sigma \phi$ is a (local) reflection and we have $\operatorname{Norm}_{\phi^{-1} \sigma \phi}(D)=$ $\left(\operatorname{Norm}_{\sigma}(D)\right)^{\phi}$.

Proof. We have $d \in\left(\operatorname{Norm}_{\sigma}(D)\right)^{\phi}$ if and only if $d^{\phi^{-1}} \in \operatorname{Norm}_{\sigma}(D)$, which is equivalent to $d^{\phi^{-1} \sigma}=\left(d^{\phi^{-1}}\right)^{-1}=\left(d^{-1}\right)^{\phi^{-1}}$, whence $d^{\phi^{-1} \sigma \phi}=d^{-1}$, which in turn is equivalent to $d \in \operatorname{Norm}_{\phi^{-1} \sigma \phi}(D)$. In the same way, we can prove $\operatorname{Fix}_{\phi^{-1} \sigma \phi}(V)=$ $\operatorname{Fix}_{\sigma}(V)^{\phi}$, and in our case that set is empty. Since $\phi$ is a graph automorphism, the graph $\Gamma_{\phi^{-1} \sigma \phi}=\left(V, D_{\phi^{-1} \sigma \phi}, \iota_{\phi^{-1} \sigma \phi},-1_{\phi^{-1} \sigma \phi}\right)$ is not connected, so $\phi^{-1} \sigma \phi$ is a (local) reflection.

Let $\Gamma$ be a graph and let $\alpha: W \rightarrow \operatorname{Aut}(\Gamma)$ be a group action on $\Gamma$. The action $\alpha$ is free if no vertex of $\Gamma$ is fixed by a group element other than the identity. This implies that $\alpha$ is injective, so we may think of $W$ as a subgroup of $\operatorname{Aut}(\Gamma)$.

Lemma 4.5. Let $\Gamma=(V, D, \iota,-1)$ be a graph and let $W$ act freely on $\Gamma$. We have

$$
\operatorname{Norm}_{w}(D) \cap \operatorname{Norm}_{v}(D)=\emptyset
$$

for each pair $v, w$ of distinct involutions in $W$. In particular, any involution $r \in W$ acting as a local reflection of $\Gamma$ is uniquely determined by the set $\operatorname{Norm}_{r}(D)$.

Proof. Notice $\operatorname{Norm}_{v}(D)=\operatorname{Norm}_{v^{-1}}(D)$. Suppose $d \in \operatorname{Norm}_{w}(D) \cap \operatorname{Norm}_{v}(D)$ and, thus, $d \in \operatorname{Norm}_{w}(D) \cap \operatorname{Norm}_{v^{-1}}(D)$. Since the operation of $W$ is free, the equality $\left(d^{\iota}\right)^{w v^{-1}}=\left(\left(d^{w v^{-1}}\right)^{\iota}\right)=\left(\left(d^{-1}\right)^{v^{-1}}\right)^{\iota}=d^{\iota}$ implies $w v^{-1}=1$, whence $w=v$. The second statement is clear since $\operatorname{Norm}_{r}(D)$ is not empty for a local reflection $r \in W$. 
Definition 4.6. Let $W$ be a group generated by a symmetric subset $S$, i.e., $S^{-1}=$ $S$, with $1 \notin S$. A word (on $S$ ) is a sequence $\left(s_{1}, s_{2}, \ldots, s_{n}\right)$ of elements of $S$. Let $w \in W$. The length $\ell_{S}(w)$ or simply $\ell(w)$ of $w$ (with respect to $S$ ) is the smallest integer $n \geq 0$ such that $w=s_{1} s_{2} \cdots s_{n}$ for a word $\left(s_{1}, s_{2}, \ldots, s_{n}\right)$. A reduced decomposition of $w$ (with respect to $S$ ) is a word $\left(s_{1}, s_{2}, \ldots, s_{n}\right)$ such that we have $w=s_{1} s_{2} \cdots s_{n}$ and $n=\ell(w)$.

Lemma 4.7. Let $\Gamma=(V, D, \iota,-1)$ be a graph and let $W$ act on $\Gamma$. Let $S$ be a generating subset of $W$ such that every element of $S$ acts as a reflection of $\Gamma$. Suppose there is a vertex $\mathbf{x}$ such that for every $s \in S$ there exists a $d_{\mathbf{x}, \mathbf{x}^{s}} \in D$ with $d_{\mathbf{x}, \mathbf{x}^{s}}^{\iota}=\mathbf{x}$ and $\left(d_{\mathbf{x}, \mathbf{x}^{s}}^{-1}\right)^{\iota}=\left(d_{\mathbf{x}^{s}, \mathbf{x}}\right)^{\iota}=\mathbf{x}^{s}$. Furthermore suppose for all $s, t \in S$, $w \in W$, that the relation $\mathbf{x}^{s}=\mathbf{x}^{w t w^{-1}}$ implies $s=w t w^{-1}$. Then the stabilizer $W_{x}$ is trivial.

Proof. Suppose we have $\mathbf{x}^{w}=\mathbf{x}$ for a $w \in W$ of minimal length $n \geq 1$. Take a reduced decomposition $\left(s_{1}, s_{2}, \ldots, s_{n}\right)$ of $w$ with respect to $S$. Consider the path

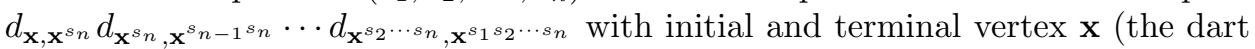
$d_{\mathbf{x}^{s_{j} \cdots s_{n}}, \mathbf{x}^{s_{j-1} s_{j} \cdots s_{n}}}$ exists as $d_{\mathbf{x}, \mathbf{x}^{s_{j-1}}}$ exists and $s_{j} \cdots s_{n}$ is an automorphism of $\Gamma$ ). The vertices $\mathbf{x}$ and $\mathbf{x}^{s_{n}}$ are separated by $s_{n}$. So there must be an index $1<$ $j \leq n$ such that $s_{n}$ separates $\mathbf{x}^{s_{j} s_{j+1} \ldots s_{n}}$ and $\mathbf{x}^{s_{j-1} s_{j} \ldots s_{n}}$. In other words, we have $\mathbf{x}^{\left(s_{j} s_{j+1} \cdots s_{n}\right) s_{n}}=\mathbf{x}^{s_{j-1} s_{j} \cdots s_{n}}$ or equivalently $\mathbf{x}^{\left(s_{j} s_{j+1} \cdots s_{n}\right) s_{n}\left(s_{j} s_{j+1} \cdots s_{n}\right)^{-1}}=\mathbf{x}^{s_{j-1}}$. By hypothesis we get

$$
\left(s_{j} s_{j+1} \cdots s_{n}\right) s_{n}\left(s_{j} s_{j+1} \cdots s_{n}\right)^{-1}=s_{j-1}
$$

or equivalently $s_{j} s_{j+1} \cdots s_{n}=s_{j-1} s_{j} \cdots s_{n-1}$, whence

$$
s_{1} s_{2} \cdots s_{n}=s_{1} s_{2} \cdots s_{j-2} s_{j} s_{j+1} \cdots s_{n-1},
$$

which is a contradiction to the minimality of $n$.

Proposition 4.8. Let $\Gamma$ be a connected graph, let $W$ act on $\Gamma$ and let $S$ be a generating subset of $W$. Suppose there is a vertex $\mathbf{x} \in V$ such that for each dart $d$ with initial vertex $\mathbf{x}$ there is an $s \in S$ with $\mathbf{x}^{s}=\left(d^{-1}\right)^{\iota}$. Then the action of $W$ on the set of vertices of $\Gamma$ is transitive.

Proof. By a straightforward induction argument.

\section{Covers of MONOpoles}

Definition 5.1. An automaton is a triple $A=(S, X, \tau)$ where $S$ is a set, the set of states, $X$ is a set, the set of inputs, and $\tau: S \times X \rightarrow S$ is a map, the transition map. (By iteration we can and sometimes will consider $\tau$ as a map from $S \times X^{*}$ into $S$, where $X^{*}$ is the monoid of all words over the alphabet $X$.) The transition semi-group $G_{A}$ of the automaton $A$ consists of the transformations $g: S \rightarrow S$ such that there exists an $x \in X^{*}$ such that $s^{g}=(s, x)^{\tau}$ for all $s \in S$. The automaton $A$ is called a group automaton if its transition semi-group $G_{A}$ is a group.

Obviously, in a group automaton the map $\tau_{x}: S \rightarrow S: s \mapsto(s, x)^{\tau}$ is a permutation of $S$ for arbitrary $x \in X$. Therefore we can add the symbol $x^{-1}$ to $X$ and define the map $\tau_{x^{-1}}$ as the inverse map of $\tau_{x}$. Then $X$ admits an involution -1 with the property $\left(\mathbf{x}^{-1}\right)^{-1}=\mathbf{x}$. If a group automaton $A=(S, X, \tau)$ satisfies $X^{-1}=X$, then 
it is called symmetric. A group automaton is called homogeneous if $s^{\tau_{x}}=s^{\tau_{y}}$ for some $s \in S$ implies $s^{\tau_{x}}=s^{\tau_{y}}$ for all $s \in S$.

The transition graph $\Gamma=(V, D, \iota,-1)$ of a symmetric group automaton $A=$ $(S, X, \tau)$ consists of

$$
\begin{aligned}
\widehat{V} & =S, \\
\widehat{D} & =S \times X, \\
\iota: D \rightarrow V & : \quad(s, x) \mapsto s, \\
-1: D \rightarrow D & : \quad(s, x) \mapsto\left((s, x)^{\tau}, x^{-1}\right) .
\end{aligned}
$$

Theorem 5.2. Any transition graph of a symmetric group automaton is a 1-cover of a monopole and vice versa.

Proof. Certainly, the canonical projection $S \times X \rightarrow X$ extends to a 1-covering of the monopole $(\{S\}, X, \bar{\iota}, \overline{-1})$ with $\bar{\iota}: X \rightarrow\{S\}$ the constant map and $\overline{-1}: X \rightarrow X$ the inversion map.

Conversely, let $\widehat{\Gamma}=(\widehat{V}, \widehat{D}, \widehat{\iota}, \widehat{-1})$ be a 1 -cover of the monopole $\Gamma=(v, D, \iota,-1)$. Then $(\widehat{V}, D, \tau)$ with $(x, d)^{\tau}$ defined as the terminal vertex in $\widehat{V}$ of the unique lift of the path $d$ of length one to a path of $\widehat{\Gamma}$ with initial vertex $x$ by Lemma 2.3 , is an automaton. It is even a group automaton, because $\tau_{d^{-1}}$ is the inverse of $\tau_{d}$. Moreover, $D^{-1}=D$ implies that the group automaton is symmetric. Obviously, $\widehat{\Gamma}$ is the transition graph of $(\widehat{V}, D, \tau)$.

An automaton $A=(S, X, \tau)$ is called strongly connected if for each pair $s, t$ of states there exists an $x \in X^{*}$ with $(s, x)^{\tau}=t$. The transition graph of a symmetric group automaton is connected if and only if the corresponding automaton is strongly connected.

Lemma 5.3. Let $\widehat{\Gamma}$ be the transition graph of a strongly connected symmetric group automaton $(S, X, \tau)$ and let $\Gamma=(v, D, \iota,-1)$ be a monopole 1 -covered by $\widehat{\Gamma}$. The 1 -covering $\widehat{\Gamma} \rightarrow \Gamma$ is regular if and only if $(S, X, \tau)$ is homogeneous and for each $x \in X$ the map $\tau_{x}$ can be extended to an automorphism of $\widehat{\Gamma}$.

Proof. Suppose for each $x \in X$ the map $\tau_{x}$ can be extended to an automorphism of $\widehat{\Gamma}$ and $(S, X, \tau)$ is homogeneous. There exists an equivalence relation on $X$ defined by $x \equiv y$ if and only if $s^{\tau_{x}}=s^{\tau_{y}}$ for some, whence by homogeneity of $(S, X, \tau)$, for all $s \in S$. Certainly $(S, X / \equiv, \bar{\tau})$ is a homogeneous strongly connected symmetric group automaton and for each $\bar{x} \in X / \equiv$ the map $\bar{\tau}_{\bar{x}}$ can be extended to an automorphism of the transition graph of $(S, X / \equiv, \bar{\tau})$. All edges except at most one of the transition graph of $(S, X / \equiv, \bar{\tau})$ are links. Moreover, each link is uniquely determined by its initial and terminal vertices. It is clear that each map $\tau_{\bar{x}}$ induces a (uniquely determined) deck transformation of the 1-covering from the transition graph of $(S, X / \equiv, \bar{\tau})$ onto some monopole with dart set $X / \equiv$. But now it is trivial to extend $\tau_{x}$ to a deck transformation of the 1-covering $\widehat{\Gamma} \rightarrow \Gamma$. The strong connectedness of $(S, X, \tau)$ yields transitivity of the group of deck transformations on the vertex fibers, whence $\widehat{\Gamma} \rightarrow \Gamma$ is regular.

Conversely, assume that $\widehat{\Gamma} \rightarrow \Gamma$ is regular. Then for each pair $\mathbf{x}, \mathbf{y}$ of neighbors in $\widehat{\Gamma}$ there exists a deck transformation mapping $\mathbf{x}$ to $\mathbf{y}$. This implies the homogeneity of $(S, X, \tau)$. Moreover, the restriction of this automorphism to the set of vertices 
coincides with the map $\tau_{d}$ where $d$ is the dart in $\Gamma$ that lifts to some dart $\widehat{d}$ in $\widehat{\Gamma}$ with initial vertex $\mathbf{x}$ and terminal vertex $\mathbf{y}$.

Example 5.4 (An inhomogeneous group automaton). Let $S=\{1,2,3,4\}$, let $X=$ $\left\{a, a^{-1}, b, b^{-1}, c, c^{-1}\right\}$ and define $\tau$ as follows:

$\begin{array}{ccccccc} & a & a^{-1} & b & b^{-1} & c & c^{-1} \\ 1 & 2 & 4 & 2 & 2 & 4 & 4 \\ 2 & 3 & 1 & 1 & 1 & 3 & 3 \\ 3 & 4 & 2 & 4 & 4 & 2 & 2 \\ 4 & 1 & 3 & 3 & 3 & 1 & 1\end{array}$.

Notice that the map $\tau_{a}$ can be extended to an automorphism of the transition graph $\Gamma$ of the group automaton $(S, X, \tau)$ but not to a deck transformation of the canonical 1-covering. Indeed, if an automorphism of $\Gamma$ induced by $\tau_{a}$ is a deck transformation, then it has to preserve the fiber $\pi^{-1}(a)$ of the 1-covering $\pi: \Gamma \rightarrow(\{S\}, X, \iota,-1)$. But then this automorphism has to interchange the fibers $\pi^{-1}(b)$ and $\pi^{-1}(c)$ and, thus, is not a deck transformation.

In 13 one can find a characterization of graphs that are regular 1-covers of monopoles. Here is a brief reminder of their result.

Definition 5.5. A voltage space on a connected graph $\Gamma$ is a triple $(F, G, \xi)$, where $G$ is a group acting on a non-empty set $F$ and $\xi: \amalg_{1}(\Gamma) \rightarrow G$ is a homomorphism of groupoids. The group $G$ is called the voltage group, the set $F$ is the abstract fiber and $[\gamma]_{1}^{\xi}$ is the voltage of the 1-homotopy class of the path $\gamma$.

In case $G$ acts on $F=G$ by right translation, then $(F, G, \xi)$ is called a Cayley voltage space.

To any voltage space $(F, G, \xi)$ over some graph $\Gamma$ there exists the associated graph $\widehat{\Gamma}=(\widehat{V}, \widehat{D}, \widehat{\iota}, \widehat{-1})$ with

$$
\begin{aligned}
\widehat{V} & =V \times F \\
\widehat{D} & =D \times F, \\
\widehat{\iota}: \widehat{D} \rightarrow \widehat{V} & : \quad(d, f) \mapsto\left(d^{\iota}, f\right), \\
\widehat{-1}: \widehat{D} \rightarrow \widehat{D} & : \quad(d, f) \mapsto\left(d^{-1}, f^{\left(d^{\xi}\right)}\right) .
\end{aligned}
$$

One can check that the canonical projection $\pi: \widehat{\Gamma} \rightarrow \Gamma$ induced by $(d, f)^{\pi}=d$ is a 1-covering.

It was observed in [13] (see also [10]) that with each regular 1-covering $\pi: \widehat{\Gamma} \rightarrow \Gamma$ one can associate a Cayley voltage space as follows. Choose $G$ to be the group of deck transformations $\Delta(\pi)$ and label the elements of each vertex fiber by $G$ so that the left action of $\Delta(\pi)$ on $\widehat{\Gamma}$ induces the action of $\Delta(\pi)$ on itself by left translation on each labelled vertex fiber. The homomorphism $\xi: \amalg_{1}(\Gamma) \rightarrow \Delta(\pi)$ is given by $d^{\xi}=g_{1}^{-1} g_{2}$ for each dart $d$ if there exists a dart in $\pi^{-1}(d)$ passing from the element labelled $g_{1}$ in the vertex fiber of $d^{\iota}$ to the element labelled $g_{2}$ in the vertex fiber of $\left(d^{-1}\right)^{\iota}$. Note that $\xi$ is well-defined. Indeed, if there exists another dart in $\pi^{-1}(d)$ with initial vertex labelled $g_{3}$ and terminal vertex labelled $g_{4}$, then, by the left action of $G=\Delta(\pi)$, we have $g_{3} g_{1}^{-1}=g_{4} g_{2}^{-1}$ and hence $g_{1}^{-1} g_{2}=g_{3}^{-1} g_{4}$.

Given a group $G$ and a generating multiset $S=S^{-1}$ of $G$ (i.e., we have a map $\alpha: S \rightarrow G$ with $\left\langle S^{\alpha}\right\rangle=G$ and $\left(s^{-1}\right)^{\alpha}=\left(s^{\alpha}\right)^{-1}$ for all $\left.s \in S\right)$, the Cayley graph Cay $(G, S)$ is the graph $(G, G \times S, \iota,-1)$, where $(g, s)^{\iota}=g$ and $(g, s)^{-1}=\left(g s^{\alpha}, s^{-1}\right)$. 
Theorem 5.6 (Malnic, Nedela, Skoviera [13]). Any Cayley graph is a regular 1cover of a monopole and vice versa.

Proof. Consider the Cayley graph $\operatorname{Cay}(G, S)$ of the group $G$ with respect to the generating multiset $S=S^{-1}$. Then the map induced by the canonical projection $G \times S \mapsto S$ extends to a regular 1-covering of the monopole $(\{G\}, S, \bar{\iota}, \overline{-1})$ with $\bar{\iota}: S \rightarrow\{G\}$ the constant map and $\overline{-1}: S \rightarrow S$ the inversion map.

To prove the converse, recall that by the above any regular 1-covering $\pi: \widehat{\Gamma} \rightarrow \Gamma$ gives rise to some Cayley voltage space $(\Delta(\pi), \Delta(\pi), \xi)$. Suppose we have a 1 covering of some monopole $\Gamma=(v, D, \iota,-1)$. Then the graph $\widehat{\Gamma}=(\widehat{V}, \widehat{D}, \widehat{\iota}, \widehat{-1})$ associated to the voltage space $(\Delta(\pi), \Delta(\pi), \xi)$ consists of

$$
\begin{aligned}
\widehat{V} & =\{v\} \times \Delta(\pi)=\Delta(\pi), \\
\widehat{D} & =D \times \Delta(\pi), \\
\widehat{\iota}: \widehat{D} \rightarrow \widehat{V} & : \quad(d, f) \mapsto\left(d^{\iota}, f\right)=(v, f)=f, \\
\widehat{-1}: \widehat{D} \rightarrow \widehat{D} & : \quad(d, f) \mapsto\left(d^{-1}, f^{\left(d^{\xi}\right)}\right)=\left(d^{-1}, f d^{\xi}\right),
\end{aligned}
$$

which is the Cayley graph of $\Delta(\pi)$ with respect to the generating multiset $D$.

Corollary 5.7. Let $\Gamma=(V, D, \iota,-1)$ be a connected graph, suppose the group $W$ acts regularly on $\Gamma$ and let $S \subseteq W$ be a symmetric, generating multiset. If for a fixed vertex $\mathbf{x}$ of $\Gamma$ the set of darts with initial vertex $\mathbf{x}$ equals $\left\{d_{s} \in D \mid s \in S\right\}$ with $\left(d_{s}^{-1}\right)^{\iota}=s . \mathbf{x}$ and $d_{s}=d_{t}$ if and only if $s=t$, then the map $\phi_{\mathbf{x}}: W \times S \rightarrow$ $D:(w, s) \mapsto w \cdot d_{s}$ induces an equivariant graph isomorphism from the Cayley graph Cay $(W, S)$ of $W$ with respect to $S$ to the graph $\Gamma$.

Proof. Let $\left(\mathbf{x},\left\{d_{s} \in D \mid s \in S\right\}, \iota^{\prime},-1^{\prime}\right)$ be a monopole, where $\iota^{\prime}:\left\{d_{s} \in D \mid s \in\right.$ $S\} \rightarrow\{\mathbf{x}\}$ is the constant map and $-1^{\prime}:\left\{d_{s} \in D \mid s \in S\right\} \rightarrow\left\{d_{s} \in D \mid s \in\right.$ $S\}: d_{s} \mapsto d_{s^{-1}}$. The map $\pi:(V, D, \iota,-1) \rightarrow\left(\mathbf{x},\left\{d_{s} \in D \mid s \in S\right\}, \iota^{\prime},-1^{\prime}\right)$ with $\left(w \cdot d_{s}\right)^{\pi}=d_{s}$ is a regular 1-covering. Indeed, it is a morphism as $d_{s}^{-1}=s . d_{s^{-1}}$ and $w \cdot d_{s}^{-1}=w s \cdot d_{s^{-1}}$ whence $(-1) \pi=\pi\left(-1^{\prime}\right)$ (the condition $\iota \pi=\pi \iota^{\prime}$ is satisfied trivially), it is a 1-covering since the $w \cdot d_{s}, s \in S$, are the darts with initial vertex $w . \mathbf{x}$, and it is regular as the group $W$ is a lift of the identity acting regularly on $\Gamma$. In particular, by Proposition 2.4. we have $W \cong \Delta(\pi)$. Hence, by the theorem, $(V, D, \iota,-1)$ can be considered as the Cayley graph of $\Delta(\pi) \cong W$ with respect to $S$. The equivariance follows by the action $W \rightarrow \operatorname{Aut}(\operatorname{Cay}(W, S))$ : $g \mapsto\{(w, s) \mapsto(g w, s)\}$ on the Cayley graph and the action $W \rightarrow \operatorname{Aut}(\Gamma): g \mapsto$ $\left\{w . d_{s} \mapsto g w . d_{s}\right\}$ on $\Gamma$.

Remark 5.8. Consider the action of $W$ on its Cayley graph with respect to $S$, and suppose the elements of $S$ act as reflections. Due to Lemma 4.4 the set $R:=\left\{w s w^{-1}: s \in S, w \in W\right\}$ consists of reflections.

Corollary 5.9 (of Theorem 5.6). Any Cayley graph $(G, G \times S, \iota,-1)$ of a group $G$ generated by a multiset $S$ consisting of involutions is a regular 1-cover of the monopole $\left(\{G\}, S, \iota^{\prime},-1^{\prime}\right)$ (with $\iota^{\prime}$ the constant map and $-1^{\prime}$ the identity map) admitting only semi-edges. Conversely, if $\pi: \Gamma \rightarrow(v, D, \iota,-1)$ is a regular 1covering onto a monopole admitting only semi-edges, then $\Gamma$ is the Cayley graph of a group $G \cong \Delta(\pi)$ generated by involutions.

Corollary $\mathbf{5 . 1 0}$ (of Lemma 5.3 and Theorem [5.6). The transition graph $\Gamma$ of some strongly connected symmetric group automaton $(S, X, \tau)$ is a Cayley graph if and 
only if $(S, X, \tau)$ is homogeneous and for each $x \in X$ the map $\tau_{x}$ can be extended to an automorphism of $\Gamma$.

\section{Chambers}

For the next definition recall that $R=\left\{w s w^{-1}: s \in S, w \in W\right\}$.

Definition 6.1. Let $\Gamma$ be a connected graph and let $W$ act freely on $\Gamma$. Moreover, assume $W$ is generated by a subset $S$ of elements that act as reflections on $\Gamma$. Since the action of $W$ is free the elements of $S$ are involutions, so $S$ is symmetric, i.e., $S^{-1}=S$. Define the equivalence relation $\sim:=\bigcap_{s \in R} \sim_{s}$ on $X$. The equivalence classes of this relation are called chambers. Define the chamber graph $\Gamma_{C}$ on the chambers of $\Gamma$ with darts $d_{C, C^{\prime}}$ with initial vertex $C$ and terminal vertex $C^{\prime}$ for distinct chambers $C, C^{\prime}$ if there exists a dart $d$ of $\Gamma$ with $d^{\iota}=\mathbf{x} \in C$ and $\left(d^{-1}\right)^{\iota}=\mathbf{x}^{\prime} \in C^{\prime}$. Moreover, define $\left(d_{C, C^{\prime}}\right)^{-1}=d_{C^{\prime}, C}$. The group $W$ also acts on $\Gamma_{C}$, and the elements of $S$ act as reflections on $\Gamma_{C}$. Indeed, for each $s \in S$, the graph $\Gamma_{s}$ (cf. the definition of a reflection at the beginning of Section 4) consists of two connected components, and any chamber is completely contained in one component.

For $s \in R$, we shall call $\operatorname{Norm}_{s}(D)$ a wall of a chamber $C$ if $\operatorname{Norm}_{s}(D)$ contains a dart whose initial vertex is contained in $C$. By $S_{C}$ we denote the set of all $s \in R$ for which $\operatorname{Norm}_{s}(D)$ is a wall of the chamber $C$. With $S$ also $R$ consists of involutions, and so does $S_{C} \subseteq \tilde{S}$.

Let $s \in R$. We say that a path $d_{C_{0}, C_{1}} d_{C_{1}, C_{2}} \cdots d_{C_{n-1}, C_{n}}$ crosses $s$ if the set of indices $0 \leq j<n$ with $C_{j} \chi_{s} C_{j+1}$ is not empty. The wall $s$ is crossed $n$ times by a path if the set of indices $0 \leq j<n$ with $C_{j} \chi_{s} C_{j+1}$ has $n$ elements.

Lemma 6.2. If the dart $d_{C, C^{\prime}}$ exists, then there is a unique element $s \in R$ separating $C$ and $C^{\prime}$. For this element we have $C^{s}=C^{\prime}$.

Proof. Since $d_{C, C^{\prime}}$ is a dart of the chamber graph, there exists a dart $d$ of $\Gamma$ with $d^{\iota}=\mathbf{x} \in C$ and $\left(d^{-1}\right)^{\iota}=\mathbf{x}^{\prime} \in C^{\prime}$. Since $C$ and $C^{\prime}$ are distinct, there is an $s \in S$ separating $\mathbf{x}$ and $\mathbf{x}^{\prime}$. This means $d \in \operatorname{Norm}_{s}(D)$, so we have $\mathbf{x}^{s}=\mathbf{x}^{\prime}$ and hence $C^{s}=C^{\prime}$. For any reflection $r$ separating $\mathbf{x}$ and $\mathbf{x}^{\prime}$ we get $d \in \operatorname{Norm}_{r}(D)$ and can conclude $s=r$ due to Lemma 4.5, which proves the uniqueness of $s$.

Recall that $W$ acts freely on $\Gamma$.

Proposition 6.3. The distance between two chambers $C$ and $C^{\prime}$ is equal to the number of elements $s \in R$ separating $C$ and $C^{\prime}$.

Proof. Let $C$ and $C^{\prime}$ be chambers and let $d_{C, C_{1}} d_{C_{1}, C_{2}} \cdots d_{C_{n-1}, C^{\prime}}$ be a minimal path from $C$ to $C^{\prime}$ of length $n$. Let $m$ be the number of elements $s \in R$ separating $C$ and $C^{\prime}$. For every $s \in R$ separating $C$ and $C^{\prime}$ there is an index $0 \leq j<n$ such that $s$ separates $C_{j}$ and $C_{j+1}$ and $s$ is uniquely determined by this property due to Lemma 6.2. This entails $m \leq n$.

Now we assume $m<n$. So there is an $s \in R$ separating $C$ and $C^{\prime}$ such that there are two different indices $j$ and $j^{\prime}$ with $C_{j} \chi_{s} C_{j+1}$ and $C_{j^{\prime}} \chi_{s} C_{j^{\prime}+1}$. Without loss of generality, we can assume $j<j^{\prime}$ and $C_{j+1} \sim_{s} C_{j^{\prime}}$. But then the path $d_{C, C_{1}} \cdots d_{C_{j-1}, C_{j}=C_{j+1}^{s}} \cdots d_{C_{j^{\prime}}^{s}=C_{j^{\prime}+1}, C_{j^{\prime}+2}} \cdots d_{C_{n-1}, C^{\prime}}$ has length $n-2$, which is a contradiction to the fact that the path is minimal.

Corollary 6.4. Minimal galleries cross every $s \in R$ at most once. 
Proposition 6.5. The group $W$ is generated by $S_{C}$ and acts transitively on the set of chambers, and $R$ is the set of all reflections in $W$.

Proof. By Proposition 4.8 the subgroup $\tilde{W}:=\left\langle S_{C}\right\rangle$ of $W$ generated by the elements of $S_{C}$ acts transitively on the set of chambers. Since $R$ consists of reflections and generates $W$, it suffices to show that every reflection lies in $\tilde{W}$. Let $r$ be a reflection and let $d \in \operatorname{Norm}_{r}(D)$. Then $d^{\iota}=\mathbf{x}_{1}$ lies in a chamber $\tilde{C}$. There is a $w \in \tilde{W}$ such that $C^{w}=\tilde{C}$. Since $\operatorname{Norm}_{r}(D)$ is a wall of $\tilde{C}$, the set $\operatorname{Norm}_{r}(D)^{w^{-1}}=$ $\operatorname{Norm}_{w r w^{-1}}(D)$ (cf. Lemma 4.4) is a wall of $C$. We get $w r w^{-1} \in S$. This means that $r \in \tilde{W}$ and $r \in R$. Since $R$ consists of reflections, it is precisely the set of all reflections. Since it generates $W$ and its elements lie in $\tilde{W}$, the set $S_{C}$ generates $W$.

Proposition 6.6. The group $W$ acts regularly on the set of chambers.

Proof. Let $C$ be a chamber. Then $d_{C, C^{s}}$ is a dart of the chamber graph for every $s \in S_{C}$. If we have $C^{\tilde{s}}=C^{s}$ for $s \in S_{C}$ and $\tilde{s} \in R$, then we get $\tilde{s}=s$ by Lemma 6.2 . So by Lemma 4.7 the stabilizer $W_{C}$ is trivial. Since $W$ acts transitively on the set of chambers, this implies regularity.

Corollary 6.7. The chamber graph $\Gamma_{C}$ is isomorphic to the Cayley graph Cay $\left(W, S_{C}\right)$. Moreover, this isomorphism is $W$-equivariant.

Proof. This follows by the proposition and Corollary 5.7 .

Corollary 6.8. There exists a 1-1 correspondence between words on $S$ and paths in $\Gamma_{C}$ with initial vertex $C$. Two words on $S$ represent the same element of $W$ if and only if the terminal vertices of the corresponding paths based at $C$ coincide.

The following result states for a very special case that $S_{C}$ is a minimal generating set for $W$. We will need this result for the proof of Theorem 7.4 .

Lemma 6.9. Let $C$ be a chamber and let $s_{1} \neq s_{2} \in S_{C}$. Suppose that $W$ is generated by $S=\left\{s_{1}, s_{2}\right\}$. Then $S=S_{C}$.

Proof. According to [2, Ch. IV $\S 1$ ], the pair $(W, S)$ is a Coxeter system, as $S$ consists of two involutions. Let $\Gamma_{1}$ be the Cayley graph of $W$ with respect to $S$, and let $\Gamma_{2}$ be the Cayley graph of $W$ with respect to $S_{C}$. Both graphs have the same set of vertices, and every dart in $\Gamma_{1}$ is a dart in $\Gamma_{2}$. By Corollary 6.7, the chamber graph can be identified with $\Gamma_{2}$. It is easily verified by studying the Cayley graph of dihedral groups (i.e., groups generated by two involutions) that, if we add a non-empty set of edges to the graph $G_{1}$, then the resulting graph is not $G$-invariant or the group elements $\left\{w^{-1} s w: w \in W, s \in S\right\}$ do not all act as reflections on it. We conclude that both graphs $\Gamma_{1}$ and $\Gamma_{2}$ have the same set of darts. Since the number of darts emanating from 1 in the Cayley graph is just the number of generators, we obtain $|S|=\left|S_{C}\right|$, and thus $S_{C}=S$.

The following result shows an interesting way of characterizing the finiteness of the group $W$.

Definition 6.10. Let $C$ be a chamber. Set $-C:=\bigcap_{s \in R} X_{-}^{s}(C)$, so $-C$ is the (possibly empty) set of all vertices, which are separated by every $s \in R$ from the vertices in $C$. 
Theorem 6.11. Let $C$ be a chamber. The group $W$ is finite if and only if the set $-C$ is non-empty; in this case $-C$ is a chamber. If $W$ is finite, then the following hold:

(i) The map $C \mapsto-C$ is an equivariant involution of the chamber graph.

(ii) $S_{C}=S_{-C}$.

(iii) $-C=\bigcap_{s \in S_{C}} X_{-}^{s}(C)$.

(iv) The chamber $-C$ is the unique chamber with maximal distance from the chamber $C$.

Proof. Assume that $-C$ is non-empty and let $\mathbf{x}, \mathbf{y} \in-C$. The vertices $\mathbf{x}, \mathbf{y}$ cannot be separated by any $s \in R$, as $\mathbf{x}, \mathbf{y}$ both are separated by all $s \in R$ from any $\mathbf{z} \in C$. Therefore $-C$ is a chamber. All elements of $R$ separate $C$ and $-C$. The chamber graph is connected, because the chambers partition the vertex set of $\Gamma$ and $\Gamma$ is connected, so by Proposition 6.3 the set $R$ is finite. As a consequence, the set of all chambers is finite. Since $W$ acts regularly on this set, it is a finite group.

Now let $W$ be finite. So the set of chambers is finite. Let $\tilde{C}$ be a chamber with maximal distance $n$ from $C$. Suppose there is an $s \in R$ that does not separate $\tilde{C}$ and $C$. Then the chamber $\tilde{C}^{s}$ has distance $m \leq n$ from $C$. Let $d_{C, C_{1}} \cdots d_{C_{m-1}, C_{m}=\tilde{C}^{s}}$ be a minimal path from $C$ to $\tilde{C}^{s}$. There is an index $0 \leq j<m$ such that $C_{j}^{s}=C_{j+1}$. The path $d_{C, C_{1}} \cdots d_{C_{j}=C_{j+1}^{s}, C_{j+2}^{s}} \cdots d_{C_{m-1}^{s}, C_{m}^{s}=\tilde{C}}$ has length $m-1<n$, which is a contradiction. So we have $\tilde{C}=-C$. We have proved the first assertion and part (iv).

To prove (i) let $m$ be the distance between $C$ and $-C$. Since $W$ acts transitively on the chamber graph, for any chamber $\tilde{C}$ the distance of any chamber $\tilde{C}$ and $-\tilde{C}$ is equal to $m$. So the given map is an involution. Now let $w \in W$ and let $C$ be a chamber. Then the distance between $C^{w}$ and $(-C)^{w}$ equals the distance $m$ between $C$ and $-C$, which entails $-\left(C^{w}\right)=(-C)^{w}$. So the map is equivariant. If two chambers $C$ and $\tilde{C}$ are adjacent, then the distance between $-C$ and $\tilde{C}$ is $m-1$. Thus $-C$ and $-\tilde{C}$ are separated by one and only one element of $R$. So they are adjacent.

For part (ii) let $C$ be a chamber. Then $\operatorname{Norm}_{s}(D)$ is a wall of $C$ if and only if $C$ and $C^{s}$ are adjacent. This is equivalent to $-C$ and $-C^{s}$ being adjacent.

Finally, we prove (iii). Suppose $C$ and $C^{\prime}$ are separated by every element of $S_{C}$. Now let $\tilde{s} \in R$ and $s \in S_{C}$. Suppose $\tilde{s}$ separates $C$ and $C^{\prime}$. Since $S_{C}$ generates $W$, it suffices to show that $s \tilde{s} s^{-1}$ separates $C$ and $C^{\prime}$. Certainly $s \tilde{s} s^{-1}$ separates $C^{s}$ and $C^{\prime s}$. If $s \tilde{s} s^{-1}=s$, then $\tilde{s}=s$ and we are done. Otherwise, recall that $s$ is the only element of $R$ which separates $C$ and $C^{s}$. The same is true for $C^{\prime}$ and $C^{\prime s}$. So neither $C$ and $C^{s}$ nor $C^{\prime}$ and $C^{\prime s}$ are separated by $\tilde{s}$. This means $\tilde{s}$ separates $C^{s}$ and $C^{\prime s}$ and, thus, $s \tilde{s} s^{-1}$ separates $C$ and $C^{\prime}$.

Corollary 6.12 (of Corollary 6.8 and Theorem 6.11). If $W$ is finite, there exists a unique longest element $w_{0}$ in $W$ with respect to $S$ and it has length $|R|$.

For the case of dihedral groups we will need the statement of Theorem 6.11(iii) but without requiring $W$ to be finite:

Lemma 6.13. If $S_{C}=\left\{s_{1}, s_{2}\right\}$, then

$$
-C=\bigcap_{s \in S_{C}} X_{-}^{s}(C) .
$$


Proof. If the set $D:=\bigcap_{s \in S_{C}} X_{-}^{s}(C)$ is empty, then so is $-C$ by definition. So we suppose that $D$ is not empty and deduce that $W$ is finite. Then we are done by Theorem 6.11 (iii). So let $\tilde{C}$ be a chamber in $D$ with minimal distance $m$ to $C$. Recall the statement of Proposition 6.3. The distance between $C^{s_{1}}$ and $\tilde{C}$ is $m-1$. So there is a path of length $m-1$ starting with the dart $d_{\tilde{C}, C^{\prime}}$ and ending in $C^{s_{1}}$. The chamber $C^{\prime}$ must lie outside of $D$ due to the minimality of the distance between $C$ and $\tilde{C}$. The path does not cross $s_{1}$, so the path $d_{\tilde{C}, C^{\prime}}$ must cross $s_{2}$. Thus $\operatorname{Norm}_{s_{2}}(D)$ is a wall of $\tilde{C}$. By swapping the roles of $s_{1}$ and $s_{2}$ we see that $E^{s_{1}}$ is also a wall of $\tilde{C}$. Due to the transitive action of $W$ on chambers, every chamber has two walls. We obtain $D=\tilde{C}$. Now we can proceed as in the proof of Theorem 6.11 (iii).

\section{Coxeter groups}

Definition 7.1. Let $W$ be a group generated by a subset $S$ of proper involutions. For $s$ and $t \in S$ let $m(s, t) \in \mathbb{N} \cup \infty$ be the order of st in $W$. Set $I:=\{(s, t) \in$ $S \times S \mid m(s, t)<\infty\}$. The pair $(W, S)$ is called a Coxeter system if $W$ has a presentation of the form $W=\langle S|(s t)^{m(s, t)}=1$ for all $\left.(s, t) \in I\right\rangle$. Then the group $W$ is called a Coxeter group.

In Section 8 we want to relate Coxeter groups to the Cayley graphs and covers of monopoles from Section 5. Therefore we have to allow for repetition of generators and trivial generators in Coxeter groups. More precisely, if $W$ is a Coxeter group with respect to a generating system $S \subset W$ of involutions and $\alpha: D \rightarrow S \cup\left\{1_{W}\right\}$ is a surjection, then we call $W$ a Coxeter group with respect to the generating multiset $D$. In view of the map -1 in a graph we set $d^{-1}:=d$ for each $d \in D$. The relation $\equiv$ from the proof of Lemma 5.3 shows that one can pass between the two notions without a problem.

Remark 7.2. The existence of a presentation of the form in the above definition is equivalent to either of the following conditions:

(i) The system $(W, S)$ satisfies the following universal property: Let $G$ be a group and let $f: S \rightarrow G$ be a map with $(f(s) f(t))^{m(s, t)}=1$ for all $(s, t) \in I$. Then there is a unique homomorphism $\tilde{f}: W \rightarrow G$ extending $f$.

(ii) Let $\bar{W}$ be a group and let $f: \bar{W} \rightarrow W$ be a homomorphism. Assume that there is a map $h: S \rightarrow \bar{W}$ with $f(h(s))=s$ and $(h(s) h(t))^{m(s, t)}=1$ for $(s, t) \in I$ and such that the elements $h(s)$ with $s \in S$ generate $\bar{W}$. Then $f$ is injective.

Definition 7.3. We say that the pair $(W, S)$ satisfies the exchange condition if the following holds. Let $w \in W$ and $s \in S$ such that $\ell(s w) \leq \ell(w)$. Then for every reduced decomposition $\left(s_{1}, s_{2}, \ldots, s_{n}\right)$ of $w$, there is an integer $1 \leq j \leq n$ such that $s s_{1} s_{2} \cdots s_{j-1}=s_{1} s_{2} \cdots s_{j}$.

Theorem 7.4. For any chamber $C$ the pair $\left(W, S_{C}\right)$ is a Coxeter system.

Proof. Let $\bar{W}$ be a group and let $f: \bar{W} \rightarrow W$ be a homomorphism. Assume there exists a map $h: S_{C} \rightarrow \bar{W}$ with $f(h(s))=s$ and $\left(h(s) h\left(s^{\prime}\right)\right)^{m\left(s, s^{\prime}\right)}=1$ for $\left(s, s^{\prime}\right) \in I$ and such that the elements $h(s)$ with $s \in S_{C}$ generate $\bar{W}$. If we show that $f$ is injective, we are done by Remark 7.2 , 


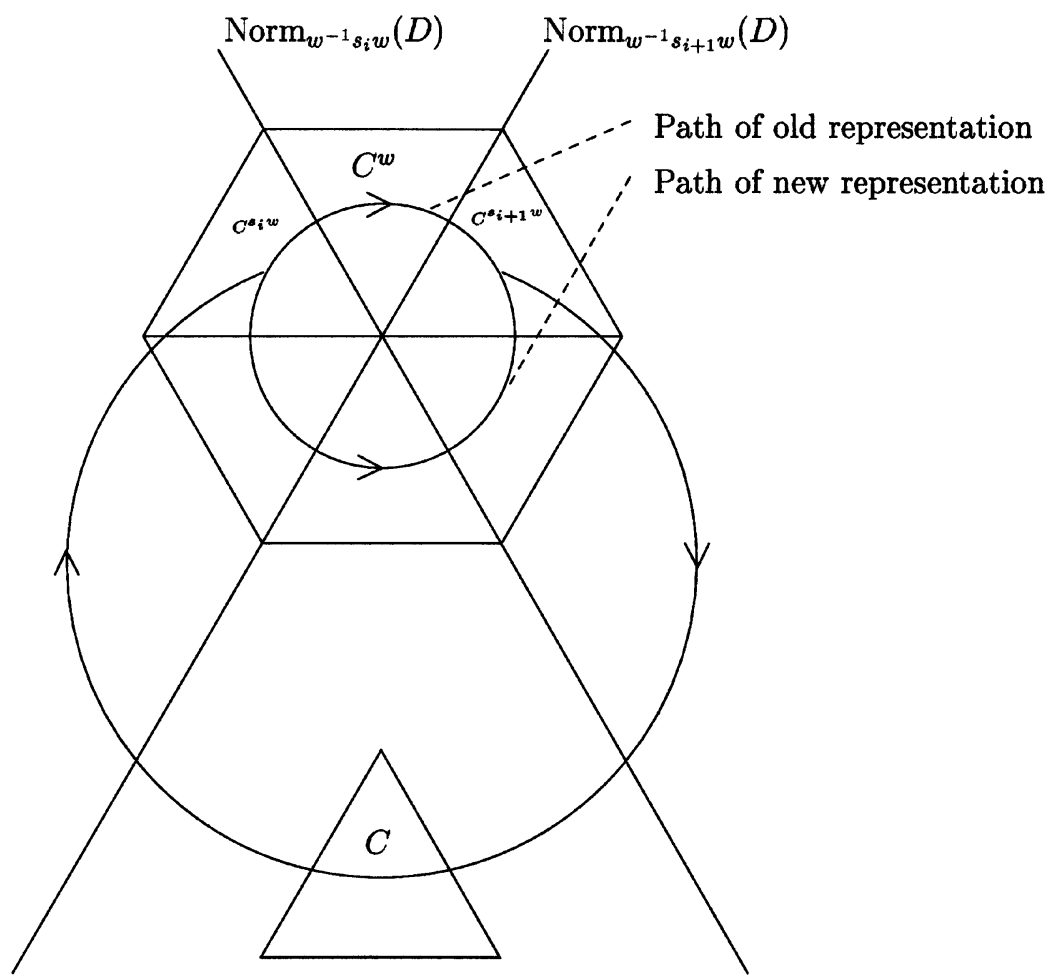

Figure 1. Paths corresponding to the old and the new representations of $g$

Since each $s \in S_{C}$ is an involution, so is each $h(s) \in \bar{W}, s \in S_{C}$, whence every element $g \in \bar{W}$ can be represented as $g=h\left(s_{n}\right) h\left(s_{n-1}\right) \cdots h\left(s_{1}\right)$ with $n \in \mathbb{N}$ and $s_{1}, s_{2}, \ldots, s_{n} \in S_{C}$. Suppose $g \in \operatorname{ker} f$ and let $C$ be a chamber. This means that

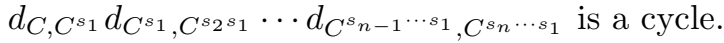

Let $k$ be the maximal value occurring as a distance between $C$ and $C^{s_{i} s_{i-1} \cdots s_{1}}$ for some $0<i<n$, and let $i$ be the maximal value for which $C^{s_{i} s_{i-1} \cdots s_{1}}$ has distance $k$ from $C$. Set $w:=s_{i} s_{i-1} \cdots s_{1}$. The chamber $C^{s_{i} w}$ is closer to $C$ than $C^{w}$. (See Figure 1.) Since $w^{-1} s_{i} w$ separates $C^{s_{i} w}$ and $C^{w}$, it also separates $C$ and $C^{w}$ by Corollary 6.4 So $s_{i}$ separates $C^{w^{-1}}$ and $C$. In the same way we show that $s_{i+1}$ separates $C^{w^{-1}}$ and $C$. Set

We now consider the subgroup $W^{\prime}$ of $W$ generated by the subset $S^{\prime}=\left\{s_{i}, s_{i+1}\right\}$.

$$
R^{\prime}:=\left\{w^{-1} s w: w \in W^{\prime}, s \in S^{\prime}\right\}
$$

Now $W^{\prime}$ is generated by the $W^{\prime}$-invariant set of reflections $R^{\prime}$. We shall call the chambers with respect to this set of reflections large chambers in order to distinguish them from the small chambers which come from the set of reflections $R$. Let $C^{\prime}$ be the large chamber that contains $C$. From Lemma 6.9 we know that $S^{\prime}=S_{C^{\prime}}$. The chamber graph with respect to $R^{\prime}$ can be identified with the Cayley graph of the 
dihedral group $W^{\prime}$ with respect to $S^{\prime}$. Since

$$
C^{w^{-1}} \subseteq \bigcap_{s \in S_{C^{\prime}}} X_{-}^{s}\left(C^{\prime}\right)
$$

the set $-C^{\prime}$ is a large chamber due to Lemma 6.13. So the group $W^{\prime}$ is finite due to Theorem 6.11. Thus the Cayley graph of $W^{\prime}$ with respect to $S^{\prime}$ is a cycle.

The large chambers $C^{\prime}$ and $-C^{\prime}$ have distance $\left|R^{\prime}\right|=m\left(s_{i}, s_{i+1}\right)$ by the definition of $-C^{\prime}$. Set

$$
\widehat{w}:= \begin{cases}\left(s_{i} s_{i+1}\right)^{\frac{m\left(s_{i}, s_{i+1}\right)}{2}} & \text { if } m\left(s_{i}, s_{i+1}\right) \text { is even } \\ \left(s_{i} s_{i+1}\right)^{\frac{m\left(s_{i}, s_{i+1}\right)-1}{2}} s_{i} & \text { if } m\left(s_{i}, s_{i+1}\right) \text { is odd. }\end{cases}
$$

Since $\widehat{w} \cdot C^{\prime}=-C^{\prime}$, the large chamber $-C^{\prime}$ contains the small chamber $\widehat{C}:=\widehat{w} \cdot C$. So the small chambers $C$ and $\widehat{C}$ have distance at least $\left|R^{\prime}\right|$.

This implies that the paths

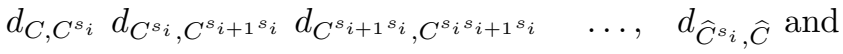

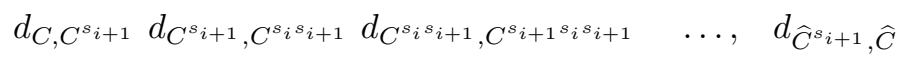

are minimal (with respect to $R$ ) and have length $\left|R^{\prime}\right|$.

Due to Theorem 6.11 (iii), the small chambers $C$ and $\widehat{C}$ are separated by each of the elements of $R^{\prime}$, and the small chambers $C^{w^{-1}}$ and $\widehat{C}$ are separated by none of the elements of $R$. Since the distance between $C$ and $C^{w^{-1}}$ is $k$, the distance between $w^{-1} . C$ and $\widehat{C}$ is $k-\left|R^{\prime}\right|$. So each of the small chambers

$$
C^{s_{i}}, C^{s_{i+1} s_{i}}, C^{s_{i} s_{i+1} s_{i}}, C^{s_{i+1} s_{i} s_{i+1} s_{i}}, \ldots, C^{s_{i+1}}
$$

has distance less than $k$ from $C^{w^{-1}}$. So the $w$-translates of these elements have distance less than $k$ from $C$.

We obtain the new representation

$$
g=h\left(s_{n}\right) h\left(s_{n-1}\right) \cdots h\left(s_{i+2}\right) \underbrace{\left(h\left(s_{i}\right) h\left(s_{i+1}\right)\right)^{m\left(s_{i}, s_{i+1}\right)-1}}_{=h\left(s_{i+1}\right) h\left(s_{i}\right)} h\left(s_{i-1}\right), \ldots, h\left(s_{1}\right) .
$$

The corresponding cyclic path has a smaller $k$ or a smaller index $i$ for which the maximal distance $k$ occurs. (See Figure 1) By induction we obtain $g=1$.

If we specialize our action of $W$ to the action on the Cayley graph, then the chambers consist of only one vertex, since the action on the points and on the chambers is simply transitive. In this case we have the following corollary of Theorem 6.11.

Theorem 7.5. Let $(W, S)$ be a Coxeter system and let $R=\left\{w s w^{-1}: s \in S, w \in\right.$ $W\}$. Then the following conditions are equivalent:

(i) $W$ is finite.

(ii) $R$ is finite.

(iii) There is a $w \in W$ such that 1 is separated from $w$ by every $s \in R$.

If condition (iii) is satisfied, then $w$ is the unique group element with maximal length and it has length $|R|$; cf. Corollary 6.12

Theorem 7.6. The following statements are equivalent:

(i) $(W, S)$ is a Coxeter system. 
(ii) $(W, S)$ satisfies the exchange condition.

(iii) The elements of $S$ act as reflections on $\operatorname{Cay}(W, S)$.

Proof. The implication (i) $\Longrightarrow$ (ii) is well-known; see e.g. [4. The implication (iii) $\Longrightarrow$ (i) is a consequence of Theorem 7.4 and Corollary 6.7

So now we assume (ii). Our proof is inspired by the ideas in [2, Ch. IV, $\S 1.7]$. Let $s \in S$ and set

$$
P_{s}:=\{w \in W: \ell(w)<\ell(s w)\} .
$$

Due to [2, Ch. IV, $\S 1.5$ Proposition 4] the group $W$ is the disjoint union of $P_{s}$ and $s . P_{s}=\{w \in W: \ell(w)>\ell(s w)\}$. Let $\left(w, s^{\prime}\right)$ be a dart with initial vertex $w \in W$ and terminal vertex $w s^{\prime}$ for $s^{\prime} \in S$. Assume $w \in P_{s}$ and $w s^{\prime} \in P_{s}^{s}$. By showing $\left(w, s^{\prime}\right) \in \operatorname{Norm}_{s}(D)$ in the Cayley graph Cay $(W, S)$, we prove that $s$ is a reflection. So we are done if we show $s w=w s^{\prime}$.

Set $n:=\ell(w)$. Due to $w \in P_{s}$, we have $\ell(s w)=n+1$. Using this and $w s^{\prime} \in P_{s}^{s}$ we get $n \leq \ell\left(s w s^{\prime}\right)<\ell\left(w s^{\prime}\right) \leq n+1$. We conclude $\ell\left(s w s^{\prime}\right)=n$ and $\ell\left(w s^{\prime}\right)=n+1$. Now let $\left(s_{1}, s_{2}, \ldots, s_{n}\right)$ be a reduced decomposition of $w$. By setting $s_{n+1}:=s^{\prime}$, we obtain the reduced decomposition $\left(s_{1}, s_{2}, \ldots, s_{n+1}\right)$ of $w s^{\prime}$. The exchange condition tells us that there is an index $1 \leq j \leq n+1$, such that we have $s s_{1} s_{2} \cdots s_{j-1}=$ $s_{1} s_{2} \cdots s_{j}$. Suppose $j<n+1$ for a moment. Then we get $s w=s s_{1} s_{2} \cdots s_{n}=$ $s_{1} s_{2} \cdots s_{j} s_{j} \cdots s_{n}=s_{1} s_{2} \cdots s_{j-1} s_{j+1} \cdots s_{n}$, which is a contradiction to $\ell(s w)=$ $n+1$. So we have $j=n+1$ and the above equation yields $s w=w s^{\prime}$.

Corollary 7.7. If $(W, S)$ is a Coxeter system, then the set $R=\left\{w s w^{-1}: s \in\right.$ $S, w \in W\}$ is exactly the subset of $W$ consisting of those elements which operate as reflections on the Cayley graph of $W$ with respect to $S$. Any subgroup generated by a subset of $R$ is a Coxeter group.

Proof. Let $(W, S)$ be a Coxeter system and consider the action of $W$ on its Cayley graph with respect to $S$. This action is free. By Proposition 6.5, the set $R$ is the set of reflections in $W$. Any subgroup of $W$ operates freely on the Cayley graph of $W$ with respect to $S$. If this subgroup is generated by reflections, then it is a Coxeter group by Theorem 7.4 .

\section{Covers of monopoles, Revisited}

Theorem 8.1. Let $\pi: \Gamma \rightarrow(v, D, \iota,-1)$ be a 1-covering of a monopole admitting semi-edges only. The graph $\Gamma$ is the Cayley graph of a Coxeter group if and only if $\pi$ is regular and any deck transformation in $\Delta(\pi)$ that interchanges two neighboring vertices of $\Gamma$ acts as a reflection on $\Gamma$.

Proof. Theorem 5.6 implies that $\Gamma$ is the Cayley graph of $\Delta(\pi)$ with respect to the generating multiset $D$ (with embedding map $\xi: D \rightarrow \Delta(\pi)$ as defined before Theorem (5.6) if and only if $\pi$ is regular. The set of vertices of $\Gamma$ equals $\Delta(\pi)$. By regularity of $\pi$ and by Lemma 4.4 it suffices to study deck transformations that interchange the vertex 1 of $\Gamma$ and some neighboring vertex $d^{\xi}$ of $\Gamma$. But any such deck transformation is given by the maps $\Delta(\pi) \rightarrow \Delta(\pi): w \mapsto d^{\xi} w$ on the vertices of $\Gamma$ and $D \times \Delta(\pi) \rightarrow D \times \Delta(\pi):(d, w) \mapsto\left(d, d^{\xi} w\right)$ on the darts. (The given map is a deck transformation interchanging 1 and $d^{\xi}$, so it has to be the one we were studying as $\Delta(\pi)$ acts regularly on $\Gamma$.) By Theorem 7.6 the group $\Delta(\pi)$ is a Coxeter group with respect to the generating multiset $D$ if and only if the elements of $D$ that do not act as the identity on $\Gamma$ act as reflections on $\Gamma$. The theorem is proved. 


\section{ACKNOWLEDGEMENT}

The authors would like to thank Arjeh Cohen, Bernhard Mühlherr, and Hendrik Van Maldeghem for discussions concerning the topics of this paper.

\section{REFERENCES}

[1] Michael A. Arbib, Theories of abstract automata, Prentice-Hall, Englewood Cliffs, 1969. MR0321626 (47:10159)

[2] Nicolas Bourbaki, Groupes et algèbres de Lie, Chapitres 4, 5 et 6, Hermann, Paris, 1968. MR0240238 (39:1590)

[3] Glen E. Bredon, Topology and geometry, Springer, Berlin, 1993. MR1224675 (94d:55001)

[4] Kenneth S. Brown, Buildings, Springer, Berlin, 1989. MR0969123 (90e:20001)

[5] Alberto Delgado, David M. Goldschmidt, Bernd Stellmacher, Groups and graphs: new results and methods, Birkhäuser, Basel, 1985. MR0862622 (88a:05076)

[6] David M. Goldschmidt, Automorphisms of trivalent graphs, Annals Math. 111 (1980), 377406. MR0569075 (82a:05052)

[7] Ralf Gramlich, Hendrik Van Maldeghem, Epimorphisms of generalized polygons. Part 1: Geometrical characterizations, Des. Codes Cryptogr. 21 (2000), 99-111. MR.1801166 (2003b:51010)

[8] Ralf Gramlich, Hendrik Van Maldeghem, Epimorphisms of generalized polygons. Part 2: Some existence and non-existence results, in: Finite Geometries, Proceedings of the Fourth Isle of Thorns Conference (edited by Aart Blokhuis, James Hirschfeld, Dieter Jungnickel, Joseph A. Thas), Kluwer Academic Publisher, Dordrecht, 2001, 177-200. MR2061805 (2005c:51003)

[9] Ralf Gramlich, Line-hyperline pairs of projective spaces and fundamental subgroups of linear groups, Adv. Geom. 4 (2004), 83-103. MR2155367(2006e:51010)

[10] Jonathan L. Gross, Voltage graphs, Discrete Math. 9 (1974), 239-246. MR0347651 (50:153)

[11] Jonathan L. Gross, Thomas W. Tucker, Topological graph theory, Wiley, New York, 1987. MR0898434 (88h:05034)

[12] Georg W. Hofmann, The geometry of reflection groups, Ph.D. thesis, TU Darmstadt, 2004.

[13] Aleksander Malnic, Roman Nedela, Martin Skoviera, Lifting graph automorphisms by voltage assignments, Europ. J. Combinatorics 21 (2000), 927-947. MR1787907 (2001i:05086)

[14] Antonio Pasini, The nonexistence of proper epimorphisms of finite thick generalized polygons, Geom. Dedicata 15 (1984), 389-397. MR0749997 (85j:51002)

[15] Herbert Seifert, William Threlfall, Lehrbuch der Topologie, Chelsea Publishing Company, New York, 1934.

[16] Jean Pierre Serre, Arbres, amalgames, $S L_{2}$, Soc. Math. France, Paris, 1977. MR0476875 $(57: 16426)$

[17] Jean Pierre Serre, Trees, Springer, Berlin, corrected second printing, 2003. MR1954121 (2003m:20032)

[18] David B. Surowski, Covers of simplicial complexes and applications to geometry, Geom. Dedicata 16 (1984), 35-62. MR0757793 (86c:57001)

TU Darmstadt, FB Mathematik / AG 5, Schlossgartenstrasse 7, 64289 Darmstadt, GERMANY

E-mail address: gramlich@mathematik.tu-darmstadt.de

TU Darmstadt, FB Mathematik / AG 5, Schlossgartenstrasse 7, 64289 Darmstadt, Germany

E-mail address: ghofmann@mathematik.tu-darmstadt.de

Current address: 5669 Merkel Street, Halifax, Nova Scotia B3K 2J1, Canada

E-mail address: hofmann@mathstat.dal.ca

TU Darmstadt, FB Mathematik / AG 5, Schlossgartenstrasse 7, 64289 Darmstadt, Germany

E-mail address: neeb@mathematik.tu-darmstadt.de 